\title{
DIRAC SUBMANIFOLDS AND POISSON INVOLUTIONS
}

\author{
BY PING XU ${ }^{1}$ \\ Dedicated to Rencontres mathématiques de Glanon on the occasion of their fifth anniversary
}

ABSTRACT. - Dirac submanifolds are a natural generalization in the Poisson category of the symplectic submanifolds of a symplectic manifold. They correspond to symplectic subgroupoids of the symplectic groupoid of the given Poisson manifold. In particular, Dirac submanifolds arise as the stable loci of Poisson involutions. In this paper, we make a general study of these submanifolds including both local and global aspects.

In the second part of the paper, we study Poisson involutions and the induced Poisson structures on their stable loci. In particular, we discuss the Poisson involutions on a special class of Poisson groups, and more generally Poisson groupoids, called symmetric Poisson groups, and symmetric Poisson groupoids. Many well-known examples, including the standard Poisson group structures on semi-simple Lie groups, Bruhat Poisson structures on compact semi-simple Lie groups, and Poisson groupoid structures arising from dynamical $r$-matrices of semi-simple Lie algebras are symmetric, so they admit a Poisson involution. For symmetric Poisson groups, the relation between the stable locus Poisson structure and Poisson symmetric spaces is discussed. As a consequence, we prove that the Dubrovin Poisson structure on the space of Stokes matrices $U_{+}$(appearing in Dubrovin's theory of Frobenius manifolds) is a Poisson symmetric space.

(c) 2003 Éditions scientifiques et médicales Elsevier SAS

RÉsumé. - La notion de sous-variété de Dirac d'une variété de Poisson est une généralisation naturelle de la notion de sous-variété symplectique d'une variété symplectique. Les sous-variétés de Dirac correspondent aux sous-groupoïdes symplectiques du groupoïde symplectique de la variété de Poisson. Une classe importante d'exemples de sous-variétés de Dirac est donnée par les points fixes d'une involution de Poisson. Nous étudions dans cet article les sous-variétés de Dirac, sous l'aspect local ainsi que sous l'aspect global.

Dans la seconde partie, nous étudions les involutions de Poisson et les structures de Poisson sur le lieu des points fixes de ces involutions. Nous étudions en particulier les involutions de Poisson sur une classe de groupes de Lie-Poisson et de groupoïdes de Poisson, les groupes et groupoïdes de Poisson symétriques. Nombre d'exemples bien connus sont symétriques et admettent donc une involution de Poisson : les structures de Lie-Poisson standard sur les groupes de Lie semi-simples, les structures de Bruhat-Poisson sur les groupes de Lie compacts semi-simples, ou encore les structures de groupoïdes de Poisson provenant d'une $r$-matrice dynamique. Dans le cas des groupes de Poisson symétriques, nous examinons le lien entre la structure de Poisson sur le lieu des points fixes et les espaces symétriques de Poisson. En corollaire, nous montrons que la structure de Poisson de Dubrovin sur l'espace $U_{+}$des matrices de Stokes (apparaissant dans la théorie de Dubrovin des variétés de Frobenius) est un espace symétrique de Poisson.

(c) 2003 Éditions scientifiques et médicales Elsevier SAS

\footnotetext{
${ }^{1}$ Research partially supported by NSF grant DMS00-72171. 


\section{Introduction}

The underlying structure of any Hamiltonian system is a Poisson manifold. To deal with mechanics with constraints, it is always desirable to understand how to equip a submanifold of a Poisson manifold with a Poisson structure. A naive way is to consider Poisson submanifolds. However, these are not very different from the original Poisson manifold from the viewpoint of the Hamiltonian systems. In addition, for symplectic manifolds, there do not exist any nontrivial Poisson submanifolds. However Dirac was able to write down a Poisson bracket for a submanifold of a symplectic manifold $P$ which is defined by a set of constraints:

$$
Q=\left\{x \in P \mid \varphi_{i}(x)=0, i=1, \ldots, k\right\}
$$

such that the matrix $\left(\left\{\varphi_{i}, \varphi_{j}\right\}\right)$ is invertible on $Q$. This is the famous Dirac bracket [8]. In this case, $Q$ is a symplectic submanifold, i.e., the pull back on $Q$ of the symplectic form is nondegenerate.

Many attempts at generalizing the Dirac brackets have been made, for example, the notion of cosymplectic manifolds of Weinstein [34], Poisson reduction of Marsden and Ratiu [29], just to name a few. In particular, Courant presented a unified approach to this question by introducing the notion of Dirac structures [6], by which one could obtain a Poisson bracket on admissible functions on a submanifold $Q$. In some cases, one can indeed obtain a Poisson structure on all functions on $Q$. Then $Q$ becomes a Poisson manifold itself.

In his study of Frobenius manifolds, which is related to the 2-dimensional topological quantum field theories, Dubrovin recently found a Poisson structure on $U_{+}$, the space of upper triangular matrices with ones on the diagonal, by viewing it as a space of Stokes matrices. Indeed Dubrovin identifies $U_{+}$with the local moduli space of semisimple Frobenius manifolds. In particular, an explicit formula was found for the Poisson bracket in the three-dimensional case (Formula F21, p. 243, [11]):

$$
\begin{gathered}
\left(\begin{array}{ccc}
1 & x & y \\
0 & 1 & z \\
0 & 0 & 1
\end{array}\right), \\
\{x, y\}=x y-2 z, \quad\{y, z\}=y z-2 x, \quad\{z, x\}=z x-2 y .
\end{gathered}
$$

This Poisson structure has various interesting properties. For instance, it naturally admits a braid group action. The Casimir function is the Markoff polynomial $x^{2}+y^{2}+z^{2}-x y z$. Its linear and quadratic parts give rise to a bi-Hamiltonian structure, etc. Then Ugaglia extended Dubrovin's formula, Eq. (3), to the $n \times n$ case [33]. Later Boalch [2] related this Dubrovin Poisson structure on $U_{+}$to the stable locus Poisson structure on the dual Poisson group $G^{*}(G=S L(n, \mathbb{C})$; see Example 5.11).

From a completely different angle, and independently, Bondal discovered the same Poisson structure on $U_{+}$in his study of triangulated categories [3]. He also extensively studied this Poisson structure, including the braid group action and symplectic leaves, etc. Bondal, in his approach, instead of writing down the Poisson structure on $U_{+}$, first discovered a symplectic groupoid $\mathcal{M}$ whose space of objects is $U_{+}$. The general theory of symplectic groupoids [35] then implies that $U_{+}$is a Poisson manifold. What is more interesting is that in a subsequent paper [4], he discovered an extremely simple relationship between his symplectic groupoid $\mathcal{M}$ and the symplectic groupoid $\Gamma$ over the Poisson group $G^{*}$ of Lu and Weinstein [26]. Namely, $\mathcal{M}$ is simply a symplectic subgroupoid of $\Gamma$ which can be realized as the stable locus of an involutive symplectic groupoid automorphism of $\Gamma$. 
Bondal's work suggests a simple fact, which was somehow overlooked in the literature: a submanifold of a Poisson manifold inherits a natural Poisson structure if it can be realized as the base space of a symplectic subgroupoid of the symplectic groupoid of the Poisson manifold. A natural question arises as to what these submanifolds are and how they can be characterized. The first aim of this paper is to answer this question. Such submanifolds will be called Dirac submanifolds. Symplectic subgroupoids are very simple to describe: they are subgroupoids and at the same time symplectic submanifolds. In contrast, Dirac submanifolds are not so simple as we shall see. There is some interesting and rich geometry there, both global and local, which we believe deserves further study.

Dirac submanifolds are a special case of the submanifolds, on which the algebra of admissible functions for the pulled-back Dirac structure is comprised of all functions [6,29]. In other words, the intersection of a Dirac submanifold with the symplectic leaves of $P$ are symplectic submanifolds of the leaves. This feature explains the existence of an induced Poisson structure on a Dirac submanifold. However, not all submanifolds satisfying this property are Dirac submanifolds. For instance, symplectic leaves (except for the zero point) of $\mathfrak{s u}(2)$ are not Dirac submanifolds (see Example 2.17). It is still not clear at the moment how to describe the global obstruction in general. On the other hand, when the Poisson manifold is symplectic, the Dirac submanifolds are precisely the symplectic submanifolds. Other examples include cosymplectic submanifolds and stable loci of Poisson involutions.

The second aim of the paper is to study systematically Poisson involutions and the induced Poisson structures on stable loci. When the underlying Poisson manifolds are Poisson groups or more generally Poisson groupoids, there is an effective way of producing Poisson involutions, namely by means of their infinitesimal invariants: Lie bialgebras, or more generally Lie bialgebroids. They are called symmetric Poisson groupoids and symmetric Lie bialgebroids. As we shall see, such a Poisson involution exists in almost every well-known example of Poisson group or Poisson groupoid, including the standard Poisson group structures on semi-simple Lie groups, Bruhat-Poisson structures on compact semi-simple Lie groups, and Poisson groupoid structures arising from dynamical $r$-matrices of semi-simple Lie algebras. For Poisson groups, such involutions were also studied by Fernandes $[14,15]$. It turns out that the induced Poisson structure on the stable locus $Q$ of the Poisson involution of a symmetric Poisson group is closely connected with Poisson symmetric spaces. In particular, we prove that the identity connected component of $Q$ is always a Poisson symmetric space. As a consequence, we prove that the space of Stokes matrices $U_{+}$with the Dubrovin Poisson structure is a Poisson symmetric space for the Poisson group $G^{*}$.

The paper is organized as follows. In Section 2, we introduce the definition of Dirac submanifolds and study their basic properties. Local Dirac submanifolds are also introduced and their relation with transverse Poisson structures is discussed. Section 3 is devoted to the study of some further properties. In particular, we study how the modular class of a Dirac submanifold is related to that of the Poisson manifold $P$. We also study Poisson group actions on Dirac submanifolds. Finally we prove that Dirac submanifolds are indeed an infinitesimal version of the symplectic subgroupoids. In Section 4, we investigate stable loci of Poisson involutions, and study Poisson involutions on Poisson groupoids by introducing the notion of symmetric Poisson groupoid. In Section 5, we consider in particular the symmetric Poisson groups and the induced Poisson structures on stable loci. The connection with Poisson symmetric spaces is discussed.

We remark that one should not confuse the Dirac submanifolds defined here with the Dirac manifolds of Courant [6,9]. Courant's Dirac manifolds are manifolds equipped with a Dirac structure, which generalize both Poisson and presymplectic manifolds. In earlier versions of this paper, some other names for what we study here such as $Q$-submanifolds and IR-submanifolds 
were used, but we feel that neither of these names reflects the complete nature of these objects. In the end, we decided to call them Dirac submanifolds, which is both short and evocative.

\section{Dirac submanifolds}

This section is devoted to the definition and study of the general properties of Dirac submanifolds.

\subsection{Definition and properties}

Let us first introduce the definition.

Definition 2.1. - A submanifold $Q$ of a Poisson manifold $P$ is called a Dirac submanifold if the tangent bundle of $P$ along $Q$ admits a vector bundle decomposition:

$$
T_{Q} P=T Q \oplus V_{Q}
$$

such that $V_{Q}^{\perp}$ is a Lie subalgebroid of $T^{*} P$, where $T^{*} P$ is equipped with the standard cotangent bundle Lie algebroid structure. $V_{Q}$ is called a Dirac complement to $Q$.

Note that the last condition above is equivalent to the condition that $V_{Q} \subseteq T P$ be a coisotropic submanifold of the tangent Poisson manifold TP. So alternatively, we have

Proposition 2.2. - A submanifold $Q \subseteq P$ is a Dirac submanifold if and only if there is a decomposition (4) such that $V_{Q} \subseteq T P$ is a coisotropic submanifold of the tangent Poisson manifold TP.

In what follows, we will see that $Q$ naturally inherits a Poisson structure. However, $Q$ in general is not a Poisson submanifold of $P$. First we need to introduce some notations. By pr, we denote the bundle map $T_{Q} P \rightarrow T Q$ obtained by taking the projection along the decomposition (4). And we let $\mathrm{pr}^{*}: T^{*} Q \rightarrow T^{*} P$ denote the dual of $\mathrm{pr}$, by considering $T_{Q}^{*} P$ as a subbundle of $T^{*} P$. By $\mathrm{pr}_{*}$, we denote the map from $\mathfrak{X}^{d}(P)$ to $\mathfrak{X}^{d}(Q)$ naturally induced from pr, which is defined by $\operatorname{pr}_{*}(D)=\operatorname{pr}\left(\left.D\right|_{Q}\right), \forall D \in \mathfrak{X}^{d}(P)$. Here $\mathfrak{X}^{d}$ denotes the space of $d$-multivector fields.

We summarize some important properties of Dirac submanifolds in the following

THEOREM 2.3. - Let $Q$ be a Dirac submanifold of a Poisson manifold $(P, \pi)$. Then

(i) $\left.\pi\right|_{Q}=\pi_{Q}+\pi^{\prime}$, where $\pi_{Q} \in \Gamma\left(\wedge^{2} T Q\right)$ and $\pi^{\prime} \in \Gamma\left(\wedge^{2} V_{Q}\right)$;

(ii) $\pi_{Q}$ is a Poisson tensor on $Q$;

(iii) $\mathrm{pr}^{*}: T^{*} Q \rightarrow T^{*} P$ is a Lie algebroid morphism, where both $T^{*} Q$ and $T^{*} P$ are equipped with the cotangent bundle Lie algebroid structures;

(iv) for any $X \in \mathfrak{X}(P)$,

$$
\operatorname{pr}_{*}[X, \pi]=\left[\operatorname{pr}_{*} X, \pi_{Q}\right]
$$

(v) for any $x \in Q, \pi_{Q}^{\#}\left(T_{x}^{*} Q\right)=\pi^{\#}\left(T_{x}^{*} P\right) \cap T_{x} Q$;

(vi) for any $x \in Q, \pi_{Q}^{\#}\left(T_{x}^{*} Q\right)$ is a symplectic subspace of $\pi^{\#}\left(T_{x}^{*} P\right)$;

(vii) each symplectic leaf of $Q$ is the intersection of $Q$ with a symplectic leaf of $P$, which is a symplectic submanifold of that leaf.

Before proving this theorem, we need two lemmas. The following lemma, which can also be easily verified directly, follows from the fact that the natural inclusion $T Q \rightarrow T P$ is a Lie algebroid morphism. 
Lemma 2.4. - Let $Q \subseteq P$ be a submanifold. Assume that $D \in \mathfrak{X}^{d}(P)$ and $D^{\prime} \in \mathfrak{X}^{d^{\prime}}(P)$ are multi-vector fields tangent to $Q$, i.e., $\left.D\right|_{Q} \in \mathfrak{X}^{d}(Q)$ and $\left.D^{\prime}\right|_{Q} \in \mathfrak{X}^{d^{\prime}}(Q)$. Then

$$
\left.\left[D, D^{\prime}\right]\right|_{Q}=\left[\left.D\right|_{Q},\left.D^{\prime}\right|_{Q}\right]
$$

Next is the following:

Lemma 2.5. - Let $Q$ be a submanifold of a Poisson manifold $(P, \pi)$. Assume that there is a vector bundle decomposition $T_{Q} P=T Q \oplus V_{Q}$ such that $\left.\pi\right|_{Q}=\pi_{Q}+\pi^{\prime}$, where $\pi_{Q} \in \Gamma\left(\wedge^{2} T Q\right)$ and $\pi^{\prime} \in \Gamma\left(\wedge^{2} V_{Q}\right)$. Then $\pi_{Q}$ is a Poisson tensor on $Q$.

Proof. - Write $\pi^{\prime}=\sum_{i} X_{i} \wedge Y_{i}$ where $X_{i}, Y_{i} \in \Gamma\left(V_{Q}\right)$. Now let $\tilde{X}_{i}, \widetilde{Y}_{i} \in \mathfrak{X}(P)$ be (local) extensions of $X_{i}, Y_{i}$, and $\tilde{\pi}^{\prime}=\sum_{i} \tilde{X}_{i} \wedge \widetilde{Y}_{i}$. Let $\pi^{\prime \prime}=\pi-\tilde{\pi}^{\prime} \in \mathfrak{X}^{2}(P)$. Clearly $\left.\tilde{\pi}^{\prime}\right|_{Q}=\pi^{\prime}$ and $\left.\pi^{\prime \prime}\right|_{Q}=\pi_{Q}$. It follows from $[\pi, \pi]=0$ that $\left[\pi^{\prime \prime}, \pi^{\prime \prime}\right]=-2\left[\pi^{\prime \prime}, \tilde{\pi}^{\prime}\right]-\left[\tilde{\pi}^{\prime}, \tilde{\pi}^{\prime}\right]$. On the other hand, it is clear by definition that $\operatorname{pr}_{*}\left[\pi^{\prime \prime}, \tilde{\pi}^{\prime}\right]=0$ and $\operatorname{pr}_{*}\left[\tilde{\pi}^{\prime}, \tilde{\pi}^{\prime}\right]=0$. Thus $\operatorname{pr}_{*}\left[\pi^{\prime \prime}, \pi^{\prime \prime}\right]=0$. According to Lemma 2.4, the latter implies that $\left[\pi_{Q}, \pi_{Q}\right]=\operatorname{pr}_{*}\left[\pi^{\prime \prime}, \pi^{\prime \prime}\right]=0$. This concludes the proof.

Proof of Theorem 2.3. - By definition, $V_{Q}^{\perp}$ is a Lie subalgebroid of the cotangent Lie algebroid $T^{*} P$. By identifying $T^{*} Q$ with $V_{Q}^{\perp}$, one obtains a Lie algebroid structure on $T^{*} Q$, and a Lie algebroid morphism $\varphi: T^{*} Q \rightarrow T^{*} P$. Clearly, $\varphi=\mathrm{pr}^{*}$. By $\rho_{Q}$, we denote the anchor map of the Lie algebroid $T^{*} Q$. Thus we have $i \circ \rho_{Q}=\pi^{\#} \circ \varphi$, where $i: T Q \rightarrow T P$ is the natural inclusion. It follows that $\rho_{Q}=\operatorname{pr} \circ i_{\circ} \rho_{Q}=\operatorname{pr} \circ \pi^{\#} \circ \varphi=\operatorname{pr} \circ \pi^{\#} \circ \operatorname{pr}^{*}$. Hence the bundle map $\rho_{Q}: T^{*} Q \rightarrow T Q$ is skew-symmetric, and therefore defines a bivector field $\pi_{Q} \in \Gamma\left(\wedge^{2} T Q\right)$ so that $\rho_{Q}=\pi_{Q}^{\#}$. Under the decomposition (4), we have $\wedge^{2} T_{Q} P=\wedge^{2} T Q \oplus\left(T Q \wedge V_{Q}\right) \oplus \wedge^{2} V_{Q}$. It is clear that $\pi_{Q}$ is the $\Gamma\left(\wedge^{2} T Q\right)$-part of $\left.\pi\right|_{Q}$ under the above decomposition. Since $\pi^{\#}\left(V_{Q}^{\perp}\right) \subset T Q,\left.\pi\right|_{Q}$ does not involve any mixed term, i.e., the $\Gamma\left(T Q \wedge V_{Q}\right)$-part vanishes. Hence we have $\left.\pi\right|_{Q}=\pi_{Q}+\pi^{\prime}$ with $\pi^{\prime} \in \Gamma\left(\wedge^{2} V_{Q}\right)$. This proves (i).

By Lemma 2.5, $\pi_{Q}$ is indeed a Poisson tensor on $Q$. Hence (ii) follows. Next we need to show that the Lie algebroid structure on $T^{*} Q$ is indeed the cotangent Lie algebroid corresponding to the Poisson structure $\pi_{Q}$. Since $\varphi$ is a Lie algebroid morphism, $\varphi^{*}=$ pr induces a morphism of the (graded) differential algebras $\operatorname{pr}_{*}:\left(\Gamma\left(\wedge^{\bullet} T P\right), d_{* P}\right) \rightarrow\left(\Gamma\left(\wedge^{\bullet} T Q\right), d_{* Q}\right)$. Since $T^{*} P$ is the cotangent Lie algebroid, we know that $d_{* P}=[\pi, \cdot]$. To prove the claim, it suffices to show that $d_{* Q}=\left[\pi_{Q}, \cdot\right]$. To this end, given any $X \in \mathfrak{X}(Q)$, choose an extension $\widetilde{X} \in \mathfrak{X}(P)$. Write $\pi=\pi^{\prime \prime}+\tilde{\pi}^{\prime}$ as in the proof of Lemma 2.5 so that $\left.\pi^{\prime \prime}\right|_{Q}=\pi_{Q}$ and $\left.\tilde{\pi}^{\prime}\right|_{Q} \in \Gamma\left(\wedge^{2} V_{Q}\right)$. Then

$$
d_{* Q} X=\left(d_{* Q} \operatorname{pr}_{*}\right) \tilde{X}=\left(\operatorname{pr}_{*} d_{* P}\right) \tilde{X}=\operatorname{pr}_{*}[\pi, \widetilde{X}]=\operatorname{pr}_{*}\left[\pi^{\prime \prime}+\tilde{\pi}^{\prime}, \tilde{X}\right]=\operatorname{pr}_{*}\left[\pi^{\prime \prime}, \tilde{X}\right]=\left[\pi_{Q}, X\right],
$$

where the last step follows from Lemma 2.4. This proves (iii), and therefore (iv) as a consequence.

Next we prove the relation $\pi_{Q}^{\#}\left(T_{x}^{*} Q\right)=\pi^{\#}\left(T_{x}^{*} P\right) \cap T_{x} Q$. Since $i_{\circ} \pi_{Q}^{\#}=\pi^{\#} \circ \varphi$, it is obvious that $\pi_{Q}^{\#}\left(T_{x}^{*} Q\right) \subseteq \pi^{\#}\left(T_{x}^{*} P\right) \cap T_{x} Q$. Conversely, let $v \in \pi^{\#}\left(T_{x}^{*} P\right) \cap T_{x} Q$ be any vector. Then $v=\pi^{\#} \xi$ for some $\xi \in T_{x}^{*} P$. Since $T_{x}^{*} P=T_{x} Q^{\perp} \oplus V_{x}^{\perp}$, one can write $\xi=\xi_{1}+\xi_{2}$ such that $\xi_{1} \in T_{x} Q^{\perp}$ and $\xi_{2} \in V_{x}^{\perp}$. Since $V_{x}^{\perp}=\varphi\left(T_{x}^{*} Q\right), \pi^{\#} \xi_{2} \in\left(\pi^{\#} \circ \varphi\right)\left(T_{x}^{*} Q\right)=\pi_{Q}^{\#}\left(T_{x}^{*} Q\right) \subset T_{x} Q$. Hence $\pi^{\#} \xi_{1}=v-\pi^{\#} \xi_{2} \in T_{x} Q$. On the other hand, it is clear that $\pi^{\#} \xi_{1} \in V_{x}$. Hence we have $\pi^{\#} \xi_{1}=0$ and therefore $v=\pi^{\#} \xi_{2} \in \pi_{Q}^{\#}\left(T_{x}^{*} Q\right)$. We thus have the relation

$$
\pi_{Q}^{\#}\left(T_{x}^{*} Q\right)=\pi^{\#}\left(T_{x}^{*} P\right) \cap T_{x} Q .
$$

This implies that the symplectic leaves of $Q$ are the intersection of the symplectic leaves of $P$ with $Q$. 
Finally, let $D_{x}=\pi_{Q}^{\#}\left(T_{x}^{*} Q\right)$ and $D_{x}^{\prime}=\pi^{\prime \#}\left(T_{x}^{\perp} Q\right)$. It is simple to see that

$$
\pi^{\#}\left(T_{x}^{*} P\right)=D_{x} \oplus D_{x}^{\prime}
$$

and both $\pi_{Q}(x) \in \wedge^{2} D_{x}$ and $\pi^{\prime}(x) \in \wedge^{2} D_{x}^{\prime}$ are nondegenerate. Thus $\left.\left.\pi_{Q}(x)\right|_{D_{x}} ^{-1} \oplus \pi^{\prime}(x)\right|_{D_{x}^{\prime}} ^{-1}$ is the inverse of $\pi(x)$ when restricted to $\pi^{\#}\left(T_{x}^{*} P\right)$. It follows that $\pi_{Q}^{\#}\left(T_{x}^{*} Q\right)$ is indeed a symplectic subspace of $\pi^{\#}\left(T_{x}^{*} P\right)$. This implies that any symplectic leaf of $Q$ is a symplectic submanifold of a symplectic leaf of $P$. Since (vii) follows immediately from (vi), this concludes our proof of the theorem.

As an immediate consequence, we have

COROllary 2.6. - Assume that $Q$ is a Dirac submanifold of a Poisson manifold P. Then we have

(i) there is a morphism on the level of Poisson cohomology

$$
\operatorname{pr}_{*}: H_{\pi}^{*}(P) \rightarrow H_{\pi_{Q}}^{*}(Q)
$$

(ii) if $X \in \mathfrak{X}(P)$ is a vector field such that $\left.X\right|_{Q} \in \Gamma\left(V_{Q}\right)$, then $\operatorname{pr}_{*}[X, \pi]=0$.

\section{Remark 2.7. -}

(i) Although we emphasize the role of $Q$ in Definition 2.1, indeed $V_{Q}$ should be considered as "part of the structure". In other words, a Dirac submanifold should really be a pair $\left(Q, V_{Q}\right)$ since many constructions depend on the choice of $V_{Q}$. For instance, the morphism $\mathrm{pr}_{*}$ in Corollary 2.6 in general depends on the choice of $V_{Q}$.

(ii) For a given Dirac submanifold, is its Dirac complement $V_{Q}$ unique? If not, what is the relation between different choices of $V_{Q}$ ? Let $Q$ be a Dirac submanifold with a Dirac complement $V_{Q}$ and $f: P \rightarrow P$ a Poisson diffeomorphism which fixes $Q$. It is clear that $f_{*} V_{Q}$ is also a Dirac complement to $Q$. It would be interesting to study the classification of Dirac complements to a fixed Dirac submanifold $Q$ up to such an equivalence. Note that if there is a Hamiltonian diffeomorphism which fixes $Q$ and transports one Dirac complement into another, their induced morphisms $\mathrm{pr}_{*}$ in Corollary 2.6 must be identical.

(iii) Note that, according to Theorem 2.3(vii), the induced Poisson structure on a Dirac submanifold is always independent of the choice of a Dirac complement. Indeed, any submanifold $Q$ whose intersections with the symplectic leaves of $P$ are symplectic submanifolds of the leaves admits a potential Poisson tensor, which, however, might be discontinuous. This is simply the bivector field obtained by taking the inverse of the restriction of the leafwise symplectic form to $Q$. In terms of the language of Dirac structures, such submanifolds correspond precisely to those for which the pulled back Dirac structure [6] of the one corresponding to the graph of the Poisson tensor on $P$ is a bivector on each tangent space, which might be discontinuous. And even when it is smooth, so that one obtains a Poisson structure on $Q$, this submanifold $Q$ may still not be a Dirac submanifold. See Example 2.17 below.

We also note that Dirac submanifolds are a special case of the situation in [29], where general Poisson reduction was studied. This provides another route to obtain the Poisson structures on these submanifolds.

The next proposition gives an alternate definition of Dirac submanifolds, which is presumably easier to check in practice. 
Proposition 2.8. - A submanifold $Q$ of a Poisson manifold $(P, \pi)$ is a Dirac submanifold if the following conditions are all satisfied:

(i) there is a vector bundle decomposition $T_{Q} P=T Q \oplus V_{Q}$ so that $\left.\pi\right|_{Q}=\pi_{Q}+\pi^{\prime}$, where $\pi_{Q} \in \Gamma\left(\wedge^{2} T Q\right)$ and $\pi^{\prime} \in \Gamma\left(\wedge^{2} V_{Q}\right)$;

(ii) for any $X^{\prime} \in \Gamma\left(V_{Q}\right)$, there is an extension $X \in \mathfrak{X}(P)$ of $X^{\prime}$ such that $\operatorname{pr}_{*}[X, \pi]=0$.

Proof. - From (i), we know that $\pi_{Q}$ is a Poisson tensor on $Q$, and therefore $T^{*} Q$ carries a Lie algebroid structure. Hence $V_{Q}^{\perp}$, which can be naturally identified with $T^{*} Q$, is a Lie algebroid. It remains to show that this Lie algebroid structure on $V_{Q}^{\perp}$ makes it into a Lie subalgebroid of $T^{*} P$. To this end, it suffices to prove Eq. (5) for any vector field $X \in \mathfrak{X}(P)$.

If $X \in \mathfrak{X}(P)$ is such that $\left.X\right|_{Q}$ is tangent to $Q$, Eq. (5) follows from Lemma 2.4. On the other hand, assume that $\left.X\right|_{Q} \in \Gamma\left(V_{Q}\right)$. Then $\operatorname{pr}_{*}[X, \pi]=\operatorname{pr}_{*}\left[X, \pi^{\prime \prime}+\tilde{\pi}^{\prime}\right]=\operatorname{pr}_{*}\left[X, \pi^{\prime \prime}\right]$, where $\pi^{\prime \prime}$ and $\tilde{\pi}^{\prime}$ are the bivector fields introduced in the proof of Lemma 2.5. Since $\left.\pi^{\prime \prime}\right|_{Q}=\pi_{Q}$ is tangent to $Q,\left.\left[X, \pi^{\prime \prime}\right]\right|_{Q}$ depends only on $\left.X\right|_{Q}$. From assumption (ii), we thus have $\operatorname{pr}_{*}[X, \pi]=0$. This concludes the proof.

Remark 2.9. - The conditions (i) and (ii) in Proposition 2.8 can be replaced, respectively, by the following equivalent conditions:

(i) $\pi^{\#}\left(V_{Q}^{\perp}\right) \subseteq T Q$;

(ii) for any $x \in Q$, there is a set of local vector fields $X_{1}, \ldots, X_{k} \in \mathfrak{X}(P)$ around $x$ such that $\left.X_{i}\right|_{Q} \in \Gamma\left(V_{Q}\right), i=1, \ldots, k$, constitute a fiberwise basis for $V_{Q}$ and satisfy the property $\operatorname{pr}_{*}\left[X_{i}, \pi\right]=0, i=1, \ldots, k$.

Recall that the cosymplectic submanifolds of a Poisson manifold $P$ are those submanifolds $Q$ which are characterized by the two properties [34]:

(i) $Q$ intersects each symplectic leaf of $P$ transversely;

(ii) at each point of $Q$, the intersection of $T Q$ with the tangent space of the symplectic leaf is a symplectic subspace.

Lemma 2.10. - A submanifold $Q$ of a Poisson manifold $(P, \pi)$ is cosymplectic if and only if it satisfies the condition (i) in Proposition 2.8 with the property that $\pi^{\prime} \in \Gamma\left(\wedge^{2} V_{Q}\right)$ is nondegenerate.

Proof. - If $Q$ is cosymplectic, then $T_{x} P=T_{x} Q \oplus \pi^{\#}\left(T_{x} Q^{\perp}\right), \forall x \in Q$ [34]. Let $V_{Q}=\pi^{\#}\left(T Q^{\perp}\right)$. It is simple to see that $Q$ is a Dirac submanifold with $V_{Q}$ being the Dirac complement.

Conversely, assume that $Q$ is a submanifold which satisfies the condition (i) as in Proposition 2.8 with the property that $\pi^{\prime} \in \Gamma\left(\wedge^{2} V_{Q}\right)$ is non-degenerate. For any $x \in Q$, it is clear that $\pi^{\#}\left(T_{x} Q^{\perp}\right)=\pi^{\prime \#}\left(T_{x} Q^{\perp}\right) \subseteq V_{x}$. Since $\pi^{\prime}$ is non-degenerate, $\pi^{\prime \#}: T_{x} Q^{\perp} \rightarrow V_{x}$ is an isomorphism. Thus we have $V_{x}=\pi^{\#}\left(T_{x} Q^{\perp}\right)$. It follows that $T_{x} P=T_{x} Q \oplus \pi^{\#}\left(T_{x} Q^{\perp}\right)$. Hence $Q$ is cosymplectic.

\section{COROLlary 2.11. - Cosymplectic submanifolds are Dirac submanifolds.}

Proof. - Assume that $Q$ is a cosymplectic submanifold. Let $V_{Q}=\pi^{\#}\left(T Q^{\perp}\right)$. According to Lemma 2.10, it suffices to verify the last condition (ii) in Proposition 2.8. Clearly $\Gamma\left(V_{Q}\right)$ is spanned by the vector fields $\left.g X_{f}\right|_{Q}$ where $f, g \in C^{\infty}(P)$ and $f$ is constant along $Q$ (here and as well as in the sequel $X_{f}$ denotes the Hamiltonian vector field of $f$ ). Now clearly $\operatorname{pr}_{*}\left[g X_{f}, \pi\right]=\operatorname{pr}_{*}\left(X_{f} \wedge X_{g}\right)=0$, and therefore the condition (ii) in Proposition 2.8 is satisfied. This concludes the proof.

Remark 2.12. - Note that the Dirac complement to a cosymplectic submanifold must be unique. This can be seen as follows. For a Dirac submanifold with a Dirac complement $V_{Q}$, 
any other Dirac complement $V_{Q}^{\prime}$ must correspond to a bundle map $\psi: V_{Q} \rightarrow T Q$ such that $V_{Q}^{\prime}=\left\{\psi(v)+v \mid \forall v \in V_{Q}\right\}$. It is simple to see that the condition (i) in Remark 2.9 implies that $\psi_{\circ}\left(\pi^{\prime}\right)^{\#}=0$. In particular, if $Q$ is cosymplectic, $\psi$ must be zero so $V_{Q}$ is unique. However, in general, it is not clear how to elaborate the condition (ii) of Proposition 2.8 in order to give a clean description of $\psi$.

The following proposition gives a useful characterization of the Dirac submanifolds.

Proposition 2.13. - Assume that there is a set of functions $f_{1}, \ldots, f_{k} \in C^{\infty}(P)$ which defines a coordinate system on $Q$. Then $Q$ is a Dirac submanifold if

(i) the Hamiltonian vector field $X_{f_{i}}, \forall i$, is tangent to $Q$;

(ii) $d\left\{f_{i}, f_{j}\right\} \cong 0\left(\bmod d f_{i}\right)$ along $Q$.

Proof. - Let $V_{Q}=\left\{v \in T_{Q} P \mid v f_{i}=0, \forall i=1, \ldots, k\right\}$. Clearly $V_{Q}$ is a vector bundle such that $T_{Q} P=T Q \oplus V_{Q}$. Moreover $V_{Q}^{\perp}=\operatorname{span}\left\{\left.d f_{i}\right|_{Q}, i=1, \ldots, k\right\}$. Thus from (i) it follows that $\pi^{\#}\left(V_{Q}^{\perp}\right) \subseteq T Q$. Combining with (ii), we see that $V_{Q}^{\perp}$ is indeed a Lie subalgebroid of $T^{*} P$. Thus $Q$ is a Dirac submanifold.

\subsection{Examples}

Now we will discuss some examples of Dirac submanifolds. By Corollary 2.11, we already know that cosymplectic submanifolds are Dirac submanifolds. The following gives a list of other examples.

Example 2.14. - Assume that $P$ is a symplectic manifold. If $Q$ is a Dirac submanifold, then $Q$ must be a symplectic submanifold according to Theorem 2.3 (vii). On the other hand, symplectic submanifolds are automatically Dirac submanifolds since they are cosymplectic. In other words, the Dirac submanifolds of a symplectic manifold are precisely the symplectic submanifolds.

Another extreme case is the following:

Example 2.15. - If $x$ is a point where the Poisson tensor vanishes, then $\{x\}$ is a Dirac submanifold.

Example 2.16. - Let $P=\mathbb{R}^{n}$ be equipped with a constant Poisson structure. Then $P$ is a regular Poisson manifold, where the symplectic leaves are the affine subspaces $x+S$. Here $S$ is the symplectic leaf through 0 which is also a linear subspace of $\mathbb{R}^{n}$. Assume that an affine subspace $Q=u+V$, where $V$ is a linear subspace of $\mathbb{R}^{n}$, is a Dirac submanifold. Let $U$ be its Dirac complement at $u$. We have a vector space decomposition $\mathbb{R}^{n}=V \oplus U$. Theorem 2.3(i) implies that $P \cong V \times U$ as a Poisson manifold product, where $V$ and $U$ are equipped with the constant Poisson structures $\pi_{Q}(u)$ and $\pi^{\prime}(u)$ respectively. This condition is equivalent to requiring that the intersection of $V$ with $S$ be a symplectic subspace of $S$. Conversely, given any such linear subspace $V$, one can decompose $P=V \times U$ as a product of Poisson manifolds with constant Poisson structures. For $Q=V \times\{u\}$, by taking $V_{Q} \cong Q \times U$ to be constant, one easily sees that the conditions in Proposition 2.8 are indeed satisfied. Hence $Q$ is a Dirac submanifold. In conclusion, an affine Dirac submanifold is the translate of a symplectic linear subspace of $S$.

The following example, which indicates that being a Dirac submanifold is indeed a global property, was pointed out to the author by Weinstein.

Example 2.17. - Let $P=M \times C$, where each $M$-slice is a Poisson submanifold. Namely the Poisson tensor at each point $(x, t) \in M \times C$ is of the form $\pi(x, t)=\pi_{t}(x)$, where $\pi_{t}(x), t \in C$ is a family of $t$-dependent Poisson structures on $M$. Consider a particular $M$-slice $Q=M \times\left\{t_{0}\right\}$ which is a Poisson submanifold. We will investigate when $Q$ becomes a Dirac submanifold. 
Since we are only concerned with a small neighborhood of $t_{0}$ in $C$, we may identify $C$ with $\mathbb{R}^{n}$ by choosing a local coordinate system $\left(t_{1}, \ldots, t_{n}\right)$. If $Q$ is a Dirac submanifold, then the Dirac complement $V_{Q}$ must be of the form:

$$
V_{Q}=\operatorname{span}\left\{\frac{\partial}{\partial t_{i}}+X_{i} \mid i=1, \ldots, n\right\}
$$

where $X_{i}, i=1, \ldots, n$, are vector fields on $M$. Clearly the condition (i) in Proposition 2.8 is satisfied automatically. Thus according to Remark 2.9, for $Q$ to be a Dirac submanifold, it suffices that $\operatorname{pr}_{*}\left[\frac{\partial}{\partial t_{i}}+X_{i}, \pi_{t}(x)\right]=0$, for $i=1, \ldots, n$, which is equivalent to

$$
\left.\frac{\partial \pi_{t}(x)}{\partial t_{i}}\right|_{t=t_{0}}=-\left[X_{i}, \pi_{t_{0}}(x)\right], \quad i=1, \ldots, n .
$$

This means that $\left.\frac{\partial \pi_{t}(x)}{\partial t_{i}}\right|_{t=t_{0}}$ is a coboundary with respect to the Poisson cohomology operator $d_{\pi_{t_{0}}}=\left[\pi_{t_{0}}, \cdot\right]$. We thus conclude that

$Q$ is a Dirac submanifold if and only if the map $f: T_{t_{0}} C \rightarrow H_{\pi_{t_{0}}}^{2}(M): v \rightarrow\left[v\left(\pi_{t}\right)\right]$ vanishes.

Note that $v\left(\pi_{t}\right)$ is always a 2-cocycle with respect to $d_{\pi_{t_{0}}}$ because of the identity $\left[\pi_{t}, \pi_{t}\right]=0$.

As a special case, let us consider the situation where all $M$-slices are symplectic leaves. Then one obtains a map $\varphi: C \rightarrow H^{2}(M)$ by taking the symplectic class of the fiber. On the other hand, it is known that $H_{\pi_{t_{0}}}^{2}(M)$ is canonically isomorphic to $H^{2}(M)$. By identifying these two cohomology groups, we have

$$
f=-\varphi_{*} .
$$

To prove this equality, let $\omega_{t}$ denote the leafwise symplectic forms, and let $\omega_{t}^{b}: T M \rightarrow T^{*} M$ and $\pi_{t}^{\#}: T^{*} M \rightarrow T M$ be the bundle maps induced by $\omega_{t}$ and $\pi_{t}$, respectively. It follows from the equation $\omega_{t}^{b} \circ \pi_{t}^{\#}=i d$ that $\left(v\left(\pi_{t}\right)\right)^{\#}=-\pi_{t}^{\#} \circ\left(v\left(\omega_{t}\right)\right)^{b} \circ \pi_{t}^{\#}$, for any $v \in T_{t_{0}} C$. Eq. (7) thus follows immediately. Hence we conclude that a symplectic leaf $M \times\left\{t_{0}\right\}$ is a Dirac submanifold if and only if $t_{0}$ is a critical point of the map $\varphi$. For instance, the symplectic leaves in the Lie-Poisson $\mathfrak{s u}(2)$ can never be Dirac submanifolds except for the zero point.

Example 2.18. - Let $P=\mathfrak{g}^{*}$ be a Lie-Poisson structure corresponding to a Lie algebra $\mathfrak{g}$. Consider an affine subspace $Q=\mu+V$. Assume that $Q$ is a Dirac submanifold with constant Dirac complement $V_{Q}$. This amounts to saying that we have a decomposition $\mathfrak{g}=\mathfrak{l} \oplus \mathfrak{m}$ such that $V=\mathfrak{m}^{\perp}$ and $V_{Q} \cong Q \times \mathfrak{m}$ as a vector bundle over $Q$. Let $\left\{e_{1}, \ldots, e_{l}\right\}$ be a basis of $\mathfrak{l}$ and $\left\{m_{1}, \ldots, m_{t}\right\}$ a basis of $\mathfrak{m}$. Then $\left\{e_{1}, \ldots, e_{l}, m_{1}, \ldots, m_{t}\right\}$ is a basis of $\mathfrak{g}$. Now let $\left\{\lambda_{1}, \ldots, \lambda_{l}, r_{1}, \ldots, r_{t}\right\}$ denote the corresponding linear coordinates on $\mathfrak{g}^{*}$. Their Poisson brackets are given by

$$
\left\{\lambda_{i}, \lambda_{j}\right\}=\sum_{k}\left(a_{i j}^{k} \lambda_{k}+b_{i j}^{k} r_{k}\right), \quad\left\{\lambda_{i}, r_{j}\right\}=\sum_{k}\left(c_{i j}^{k} \lambda_{k}+d_{i j}^{k} r_{k}\right),
$$

where $a_{i j}^{k}, b_{i j}^{k}, c_{i j}^{k}, d_{i j}^{k}$ are constants. It is clear that $\left\{\lambda_{1}, \ldots, \lambda_{l}\right\}$ is a set of coordinate functions on $Q$ such that $V_{Q}^{\perp}$ is spanned by $d \lambda_{i}, i=1, \ldots, k$. Since $d\left\{\lambda_{i}, \lambda_{j}\right\}=\sum_{k}\left(a_{i j}^{k} d \lambda_{k}+b_{i j}^{k} d r_{k}\right)$, the condition (ii) of Proposition 2.13 implies that $b_{i j}^{k}=0$. On the other hand, we have 


$$
\begin{aligned}
\left.X_{\lambda_{i}}\right|_{Q} & =\left.\sum_{j}\left(\left\{\lambda_{i}, \lambda_{j}\right\} \frac{\partial}{\partial \lambda_{j}}+\left\{\lambda_{i}, r_{j}\right\} \frac{\partial}{\partial r_{j}}\right)\right|_{Q} \\
& =\left.\left(\sum_{j}\left\{\lambda_{i}, \lambda_{j}\right\} \frac{\partial}{\partial \lambda_{j}}+\sum_{j k}\left(c_{i j}^{k} \lambda_{k}+d_{i j}^{k} \mu_{k}\right) \frac{\partial}{\partial r_{j}}\right)\right|_{Q},
\end{aligned}
$$

where $\mu_{k}=r_{k}(\mu), k=1, \ldots, t$. It thus follows that $X_{\lambda_{i}}$ is tangent to $Q$ iff $c_{i j}^{k}=0, \forall j, k$ and $\sum_{k} d_{i j}^{k} \mu_{k}=0, \forall j$. The latter is equivalent to $\left\langle a d_{e_{j}}^{*} \mu, m_{j}\right\rangle=0$. Therefore we conclude that $\mathfrak{g}=\mathfrak{l} \oplus \mathfrak{m}$ must be a reductive decomposition (i.e., $\mathfrak{l}$ is a Lie subalgebra and $[\mathfrak{l}, \mathfrak{m}] \subseteq \mathfrak{m}$ ) and $a d_{\mathfrak{l}}^{*} \mu \in \mathfrak{m}^{\perp}$. Hence an affine Dirac submanifold of $\mathfrak{g}^{*}$ is the translate of the orthogonal of $\mathfrak{m}$ in a reductive decomposition $\mathfrak{g}=\mathfrak{l} \oplus \mathfrak{m}$, by an element $\mu$ such that $[\mathfrak{l}, \mathfrak{m}] \subset \operatorname{ker} \mu$. In this case, the induced Poisson structure can be identified with the Lie-Poisson structure on $\mathfrak{l}^{*}$.

\subsection{Local Dirac submanifolds}

We now introduce local Dirac submanifolds.

Definition 2.19. - A submanifold $Q$ of a Poisson manifold $P$ is called a local Dirac submanifold if at each point of $Q$ there is an open neighborhood which is a Dirac submanifold.

Immediately we have

Proposition 2.20.-A local Dirac submanifold naturally carries an induced Poisson structure.

Example 2.21. - If $Q$ is a symplectic leaf of $P$, by Weinstein's splitting theorem [34], locally $P \cong Q \times N$ as a product Poisson manifold. It thus follows that $Q$ is a local Dirac submanifold.

The following proposition gives a characterization of local Dirac submanifolds.

Proposition 2.22. - A submanifold $Q$ of a Poisson manifold $P$ is a local Dirac submanifold if and only if there exist local coordinates $\left(x_{1}, \ldots, x_{l}, y_{1}, \ldots, y_{t}\right)$ of $P$ at each point $q \in Q$ such that $Q$ is defined by $y_{1}=\cdots=y_{t}=0$ and the Poisson brackets of coordinate functions satisfy:

$$
\begin{aligned}
& \lambda_{i j}(x, 0)=0, \quad \forall 1 \leqslant i \leqslant l, 1 \leqslant j \leqslant t \\
& \frac{\partial \varphi_{i j}}{\partial y_{k}}(x, 0)=0, \quad \forall 1 \leqslant i, j \leqslant l, 1 \leqslant k \leqslant t,
\end{aligned}
$$

where $\varphi_{i j}(x, y)=\left\{x_{i}, x_{j}\right\}, \forall 1 \leqslant i, j \leqslant l$, and $\lambda_{i j}(x, y)=\left\{x_{i}, y_{j}\right\}, \forall 1 \leqslant i \leqslant l, 1 \leqslant j \leqslant t$.

Proof. - Assume that $Q$ is a local Dirac submanifold. For any point $q \in Q$, there exists an open neighborhood $U$ of $q$ in $P$ such that $U \cap Q$ is a Dirac submanifold. Let $V_{U \cap Q}$ be its Dirac complement. By shrinking it to a smaller neighborhood if necessary, one may always choose local coordinates $\left(x_{1}, \ldots, x_{l}, y_{1}, \ldots, y_{t}\right)$ of $U$ such that $U \cap Q$ is defined by $y_{1}=\cdots=y_{t}=0$ and $V_{U \cap Q}$ is spanned by $\left\{\frac{\partial}{\partial y_{i}} \mid i=1, \ldots, t\right\}$. In other words, $\left\{x_{1}, \ldots, x_{l}\right\}$ is a set of coordinates on $Q$ such that $V_{U \cap Q}^{\perp}$ is spanned by $\left\{d x_{i} \mid i=1, \ldots, l\right\}$. Then

$$
\left.d\left\{x_{i}, x_{j}\right\}\right|_{Q}=\sum_{k} \frac{\partial \varphi_{i j}}{\partial y_{k}}(x, 0) d y_{k}\left(\bmod d x_{i}\right) ;\left.\quad X_{x_{i}}\right|_{Q}=\sum_{j} \lambda_{i j}(x, 0) \frac{\partial}{\partial y_{j}}\left(\bmod \frac{\partial}{\partial x_{i}}\right) .
$$

It thus follows that $\lambda_{i j}(x, 0)=0,1 \leqslant i \leqslant l, 1 \leqslant j \leqslant t$ and $\frac{\partial \varphi_{i j}}{\partial y_{k}}(x, 0)=0,1 \leqslant i, j \leqslant l, 1 \leqslant k \leqslant t$.

Conversely, if such local coordinates exist in an open neighborhood $U$ of $q$ in $P$, one can verify directly that $U \cap Q$ is a Dirac submanifold by reversing the argument above. 
Remark 2.23. - It would be interesting to classify the Dirac complements of a local Dirac submanifold $Q$ up to a local Poisson diffeomorphism fixing $Q$.

The following result reveals a connection between local Dirac submanifolds and transverse Poisson structures [34].

Proposition 2.24. - If $Q$ is a local Dirac submanifold which is a cross section of a symplectic leaf $S$ at a point $q$ (i.e., $Q$ has complementary dimension to $S$ and intersects $S$ at a single point $q$ transversely), then the induced Poisson structure on $Q$, in a neighborhood of $q$, is isomorphic to the transverse Poisson structure.

Conversely, if $Q$ is a cross section of a symplectic leaf $S$ at a point $q$, then $Q$ is a Dirac submanifold in a neighborhood of $q$ and the induced Poisson structure is isomorphic to the transverse Poisson structure.

Proof. - From Weinstein's splitting theorem [34], it follows that a cross section of a symplectic leaf $S$ must be a Dirac submanifold in a small neighborhood of the intersection point. It remains to show that the induced Poisson structure on $Q$ as a Dirac submanifold is indeed isomorphic to the transverse Poisson structure.

We choose local coordinates as in the proof of Proposition 2.22. Thus $X_{x_{i}}$ are all tangent to $Q$ for $i=1, \ldots, l$. By definition, the transverse Poisson structure is $\left.\left\{x_{i}, x_{j}\right\}\right|_{Q}=\varphi_{i j}(x, 0)$, which is precisely the induced Poisson structure on $Q$ as a Dirac submanifold.

An immediate consequence, by combining with Example 2.18, is the following theorem of Molino [30].

COROLlary 2.25. - Let $\mu \in \mathfrak{g}^{*}$ and $\mathfrak{g}_{\mu}$ be the isotropy Lie algebra at $\mu$. If $\mathfrak{g}$ admits a reductive decomposition: $\mathfrak{g}=\mathfrak{g}_{\mu} \oplus \mathfrak{m}_{\mu}$, then the transverse Poisson structure at $\mu$ to the symplectic leaf $G \cdot \mu$ (i.e., the coadjoint orbit through $\mu$ ) is isomorphic to the Lie-Poisson structure on $\mathfrak{g}_{\mu}^{*}$.

\section{Properties of Dirac submanifolds}

This section is devoted to the further study of properties of Dirac submanifolds.

\subsection{Relative modular vector fields}

First we want to see how the modular class of a Dirac submanifold of $P$ is related to that of $P$. We start with the following:

Lemma 3.1.- Let $Q$ be a Dirac submanifold of a Poisson manifold $P$ with Dirac complement $V_{Q}$. Assume that $f \in C^{\infty}(P)$ satisfies the property $\left.d f\right|_{Q} \in V_{Q}^{\perp}$. Denote by $\varphi_{t}$ the flow generated by the Hamiltonian vector field $X_{f}$. Then both $T Q$ and $V_{Q}$ (hence $T Q^{\perp}$ and $V_{Q}^{\perp}$ ) are stable under $\varphi_{t}$.

Proof. - It is clear that $X_{f}$ is tangent to $Q$, and therefore $\left.\left[X_{f}, Y\right]\right|_{Q}$ is well-defined for any $Y \in \Gamma\left(T_{Q} P\right)$. If $Y \in \Gamma(T Q)$, clearly $\left.\left[X_{f}, Y\right]\right|_{Q} \in \Gamma(T Q)$. Hence $T Q$ is stable under $\varphi_{t}$.

Now assume that $Y \in \Gamma\left(V_{Q}\right)$. Let $\widetilde{Y} \in \mathfrak{X}(P)$ be any of its extensions. By the graded Jacobi identity, we have

$$
\left[X_{f}, \widetilde{Y}\right]=[[\pi, f], \widetilde{Y}]=[[f, \widetilde{Y}], \pi]-[[\widetilde{Y}, \pi], f] .
$$

Now $[[f, \tilde{Y}], \pi]=-[\tilde{Y}(f), \pi]=-\left[\tilde{Y}(f), \pi^{\prime \prime}+\tilde{\pi}^{\prime}\right]=-\left[\tilde{Y}(f), \pi^{\prime \prime}\right]-\left[\tilde{Y}(f), \tilde{\pi}^{\prime}\right]$, where $\pi^{\prime \prime}$ and $\tilde{\pi}^{\prime}$ are bivector fields on $P$ as in the proof of Lemma 2.5, i.e., $\left.\pi^{\prime \prime}\right|_{Q}=\pi_{Q}$ and $\tilde{\pi}^{\prime} \in \Gamma\left(\wedge^{2} V_{Q}\right)$. Since 
$\left.\tilde{Y}(f)\right|_{Q}=\left.Y(f)\right|_{Q}=0$, it is obvious that

$$
\left.\left[\tilde{Y}(f), \pi^{\prime \prime}\right]\right|_{Q}=\left[\tilde{Y}(f), \pi_{Q}\right]=0 .
$$

Thus $\left.[[f, \tilde{Y}], \pi]\right|_{Q} \in \Gamma\left(V_{Q}\right)$. On the other hand, according to Eq. (5), we have

$$
\operatorname{pr}_{*}[\tilde{Y}, \pi]=\left[\operatorname{pr}_{*} \tilde{Y}, \pi_{Q}\right]=0 .
$$

Therefore, one can write $\left.[\tilde{Y}, \pi]\right|_{Q}=\sum Z_{i} \wedge Z_{i}^{\prime}$ with $Z_{i}^{\prime} \in \Gamma\left(V_{Q}\right)$. Then

$$
\left.[[\tilde{Y}, \pi], f]\right|_{Q}=\sum\left(Z_{i}(f) Z_{i}^{\prime}-Z_{i}^{\prime}(f) Z_{i}\right)=\sum Z_{i}(f) Z_{i}^{\prime} \in \Gamma\left(V_{Q}\right)
$$

since $Z_{i}^{\prime}(f)=0$ by assumption. This shows that $\left.\left[X_{f}, Y\right]\right|_{Q}=\left.\left[X_{f}, \widetilde{Y}\right]\right|_{Q} \in \Gamma\left(V_{Q}\right)$, which implies that $V_{Q}$ is stable under the flow $\varphi_{t}$.

We are now ready to introduce the relative modular class. Let $\Omega^{\prime} \in \Gamma\left(\wedge^{t o p} T Q^{\perp}\right)$ be a nonzero section, which we always assume exists. Otherwise, one needs to consider densities as in [36]. For any $f \in C^{\infty}(Q)$, let $\tilde{f} \in C^{\infty}(P)$ be an extension of $f$ satisfying the property $\left.d \tilde{f}\right|_{Q} \in V_{Q}^{\perp}$. According to Lemma 3.1, the Hamiltonian flow of $X_{\tilde{f}}$ preserves both vector bundles $T Q$ and $V_{Q}$, hence it preserves $T Q^{\perp}$. It thus follows that $L_{X_{\tilde{f}}} \Omega^{\prime}$ is a section of $\wedge^{t o p} T Q^{\perp}$, and therefore $\left(L_{X_{\tilde{f}}} \Omega^{\prime}\right) / \Omega^{\prime}$ is a well-defined function on $Q$. In this way, one obtains a linear operator

$$
\nu_{r}: C^{\infty}(Q) \rightarrow C^{\infty}(Q), \quad f \rightarrow\left(L_{X_{\tilde{f}}} \Omega^{\prime}\right) / \Omega^{\prime} .
$$

Although $\nu_{r}$ appears to be a second-order operator, a simple computation, using the property that $\left.X_{\tilde{f}}\right|_{Q}$ is tangent to $Q$, shows that $\nu_{r}$ is a derivation:

$$
\nu_{r}(f g)=f \nu_{r}(g)+g \nu_{r}(f), \quad \forall f, g \in C^{\infty}(Q),
$$

and hence a vector field on $Q$; we call it the relative modular vector field corresponding to $\Omega^{\prime}$.

Let $\left(x_{1}, \ldots, x_{l}, y_{1}, \ldots, y_{t}\right)$ be the local coordinates as in Proposition 2.22. Take $\Omega^{\prime}=d x_{1} \wedge \cdots \wedge d x_{l}$. Then its relative modular vector field is

$$
\nu_{r}=\sum_{i j} \frac{\partial \lambda_{i j}}{\partial y_{j}}(x, 0) \frac{\partial}{\partial x_{i}} .
$$

Remark 3.2. - From this, one sees that, in general, $\nu_{r}$ may depend on the choice of a Dirac complement.

PROPOSITION 3.3. - $\nu_{r}$ is a Poisson vector field with respect to $\pi_{Q}$. For different choices of $\Omega^{\prime}$, the corresponding relative modular vector fields $\nu_{r}$ differ by a Hamiltonian vector field.

As a consequence, $\left[\nu_{r}\right]$ is a well defined class in the Poisson cohomology $H_{\pi_{Q}}^{1}(Q)$, which will be called the relative modular class of the Dirac submanifold $Q$. The proof of Proposition 3.3 follows from the lemma below.

Choose a nonzero section $\Omega_{Q} \in \Gamma\left(\wedge^{t o p} V_{Q}^{\perp}\right) \cong \Gamma\left(\wedge^{t o p} T^{*} Q\right)$, which we again assume exists. Then $\Omega=\Omega_{Q} \wedge \Omega^{\prime} \in \Gamma\left(\left.\wedge^{t o p} T^{*} P\right|_{Q}\right)$ is a nonzero section. Extend $\Omega$ to a volume form on $P$ (at least locally along the submanifold $Q$ ), which will be denoted by the same symbol $\Omega$. By $\nu_{P}$ and $\nu_{Q}$, we denote the modular vector fields of the Poisson manifolds $P$ and $Q$ corresponding to $\Omega$ and $\Omega_{Q}$, respectively. 
LEMMA 3.4. - The modular vector fields are related by

$$
\nu_{r}=\operatorname{pr}_{*} \nu_{P}-\nu_{Q}
$$

Proof. $-\forall f \in C^{\infty}(Q)$, let $\tilde{f} \in C^{\infty}(P)$ be an extension of $f$ satisfying the property $\left.d \tilde{f}\right|_{Q} \in V_{Q}^{\perp}$. Then $\left.L_{X_{\tilde{f}}} \Omega\right|_{Q}=\left.\nu_{P}(\tilde{f}) \Omega\right|_{Q}=\left.\left(\operatorname{pr}_{*} \nu_{P}\right)(f) \Omega\right|_{Q}$, and $\left.L_{X_{\tilde{f}}} \Omega_{Q}\right|_{Q}=\nu_{Q}(f) \Omega_{Q}$. From the derivation law $L_{X_{\tilde{f}}} \Omega=\left(L_{X_{\tilde{f}}} \Omega_{Q}\right) \wedge \Omega^{\prime}+\Omega_{Q} \wedge L_{X_{\tilde{f}}} \Omega^{\prime}$, it follows that

$$
\left(\operatorname{pr}_{*} \nu_{P}\right)(f)=\nu_{Q}(f)+\nu_{r}(f)
$$

Eq. (10) thus follows.

Another consequence, besides Proposition 3.3, is the following:

PROPOSITION 3.5. - The modular classes of the Poisson structures on $P$ and $Q$ are related by

$$
\operatorname{pr}_{*}\left[\nu_{P}\right]-\left[\nu_{Q}\right]=\left[\nu_{r}\right],
$$

where $\operatorname{pr}_{*}: H_{\pi}^{1}(P) \rightarrow H_{\pi_{Q}}^{1}(Q)$ is the morphism of Corollary 2.6.

Remark 3.6. - It would be interesting to see how other characteristic classes [7,16] on $P$ and $Q$ are related, and in particular, how to describe $\operatorname{pr}_{*}\left[C_{k}(P)\right]-\left[C_{k}(Q)\right] \in H_{\pi_{Q}}^{\bullet}(Q)$ for other characteristic class $C_{k}$.

\subsection{Poisson actions}

Next we consider Poisson group actions on Dirac submanifolds. As we shall see below, Dirac submanifolds behave well under Poisson group actions, which include the usual Hamiltonian actions as a special case.

THEOREM 3.7. - Assume that $(P, \pi)$ is a Poisson manifold which admits a Poisson action of a connected Poisson group $G$. Assume that $Q$ is a Dirac submanifold stable under the G-action. Then the action of $G$ on $Q$ is also a Poisson action. Moreover, if $J: P \rightarrow G^{*}$ is a momentum map, then $\left.J\right|_{Q}: Q \rightarrow G^{*}$ is a momentum map of the $G$-action on $Q$.

Proof. - Let $\mu_{P}: T^{*} P \rightarrow \mathfrak{g}^{*}$ and $\mu_{Q}: T^{*} Q \rightarrow \mathfrak{g}^{*}$ be the linear morphisms dual to the infinitesimal $\mathfrak{g}$-actions on $P$ and $Q$, respectively. Since the infinitesimal $\mathfrak{g}$-action on $Q: \mathfrak{g} \rightarrow \mathfrak{X}(Q)$ is the composition of the infinitesimal $\mathfrak{g}$-action on $P: \mathfrak{g} \rightarrow \mathfrak{X}(P)$ with the projection $\mathrm{pr}_{*}: \mathfrak{X}(P) \rightarrow \mathfrak{X}(Q)$, it follows that $\mu_{Q}=\mu_{P} \circ \mathrm{pr}^{*}$, where $\mathrm{pr}^{*}: T^{*} Q \rightarrow T^{*} P$ is the dual of the projection $\mathrm{pr}: T_{Q} P \rightarrow T Q$. Since $\mathrm{pr}^{*}$ is a Lie algebroid morphism according to Theorem 2.3(iii), it follows immediately from Proposition 6.1 in [38] that the $G$-action on $Q$ is also a Poisson action.

Assume that $J: P \rightarrow G^{*}$ is a momentum map for the Poisson $G$-action [24]. By definition, for any $\xi \in \mathfrak{g}, \pi^{\#}\left(J^{*} \xi^{l}\right)=\hat{\xi}$, where $\xi^{l} \in \Omega^{1}\left(G^{*}\right)$ is the left invariant one-form corresponding to $\xi$, and $\hat{\xi} \in \mathfrak{X}(P)$ is the vector field on $P$ generated by $\xi$. Thus we have $\operatorname{pr}_{*} \pi^{\#}\left(J^{*} \xi^{l}\right)=\hat{\xi}$ since $\hat{\xi}$ is tangent to $Q$. On the other hand, it is clear that $\operatorname{pr}_{*} \pi^{\#}\left(J^{*} \xi^{l}\right)=\pi_{Q}^{\#}\left(J^{*} \xi^{l}\right)$. This shows that $\left.J\right|_{Q}: Q \rightarrow G^{*}$ is indeed a momentum map for the Poisson $G$-action on $Q$.

\subsection{Symplectic subgroupoids}

Finally we consider symplectic groupoids of Dirac submanifolds. As we shall see below, Dirac submanifolds are indeed an infinitesimal version of symplectic subgroupoids: the base space of 
a symplectic subgroupoid of a symplectic groupoid is a Dirac submanifold of the base Poisson manifold of the groupoid. And conversely, this property can serve to characterize symplectic subgroupoids of symplectic groupoids.

THEOREM 3.8. - If $\Gamma^{\prime} \rightrightarrows Q$ is a symplectic subgroupoid of a symplectic groupoid $(\Gamma \rightrightarrows P, \alpha, \beta)$, then $Q$ is a Dirac submanifold of $P$. Conversely, if $P$ is an integrable Poisson manifold with symplectic groupoid $\Gamma$ and $Q$ is a Dirac submanifold whose corresponding cotangent Lie algebroid $T^{*} Q$ integrates to a Lie subgroupoid $\Gamma^{\prime}$ of $\Gamma$, then $\Gamma^{\prime}$ is a symplectic subgroupoid.

Proof. - Assume that $\Gamma^{\prime} \rightrightarrows Q$ is a symplectic subgroupoid of a symplectic groupoid $(\Gamma \rightrightarrows P, \alpha, \beta)$. By $\omega$ and $\omega^{\prime}$ we denote the symplectic forms on $\Gamma$ and $\Gamma^{\prime}$ respectively, and by $A$ and $A^{\prime}$, we denote their corresponding Lie algebroids. Then $A^{\prime}$ is a Lie subalgebroid of $A$. As vector bundles, $A \cong T_{P}^{\alpha} \Gamma$ (the subbundle of the tangent bundle of $\Gamma$ along $P$ consisting of vectors tangent to the $\alpha$-fibers) and $A^{\prime} \cong T_{Q}^{\alpha} \Gamma^{\prime}$, and the Lie algebroid morphism $A^{\prime} \rightarrow A$ is simply the inclusion $T_{Q}^{\alpha} \Gamma^{\prime} \rightarrow T_{P}^{\alpha} \Gamma$. It is well known [5] that $\omega^{b}: T_{P}^{\alpha} \Gamma \rightarrow T^{*} P$ and $\left(\omega^{\prime}\right)^{b}: T_{Q}^{\alpha} \Gamma^{\prime} \rightarrow T^{*} Q$ are isomorphisms of Lie algebroids, where $T^{*} P$ and $T^{*} Q$ are equipped with the cotangent Lie algebroids corresponding to the induced Poisson structures. Thus one obtains a Lie algebroid morphism $\varphi: T^{*} Q \rightarrow T^{*} P$ so that the following diagram

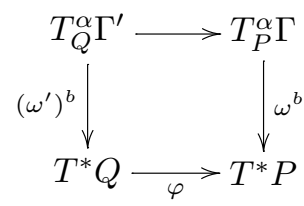

commutes. In particular, $\varphi\left(T^{*} Q\right)$ is a Lie subalgebroid of $T^{*} P$. In what follows, we will show that $\varphi^{*} \circ i$ is the identity map, where $i: T Q \rightarrow T P$ is the inclusion.

Let $\xi \in T_{x}^{*} Q$ be any covector. Assume that $\xi=\left(\omega^{\prime}\right)^{b} u$ for some $u \in T_{Q}^{\alpha} \Gamma^{\prime}$. Using the commuting diagram (12), we have for any $v \in T_{x} Q$,

$$
\left\langle\left(i^{*} \circ \varphi\right) \xi, v\right\rangle=\langle\varphi \xi, v\rangle=\left\langle\varphi\left(\omega^{\prime}\right)^{b} u, v\right\rangle=\left\langle\omega^{b} u, v\right\rangle=\omega(u, v)=\omega^{\prime}(u, v)=\left\langle\left(\omega^{\prime}\right)^{b} u, v\right\rangle=\langle\xi, v\rangle .
$$

Therefore $i^{*} \circ \varphi=i d$, or equivalently $\varphi^{*}{ }_{\circ} i=i d$. Let $V_{Q}=\operatorname{ker} \varphi^{*}$, which is a subbundle of $T_{Q} P$. Then $T_{Q} P=T Q \oplus V_{Q}$. In fact $V_{Q}^{\perp}=\varphi\left(T^{*} Q\right)$, so $V_{Q}^{\perp}$ is a Lie subalgebroid of $T^{*} P$. Hence $Q$ is a Dirac submanifold.

Conversely, assume that $Q$ is a Dirac submanifold of $P$, and $\varphi=\operatorname{pr}^{*}: T^{*} Q \rightarrow T^{*} P$ is the Lie algebroid morphism as in Theorem 2.3(iii). Let $\Gamma^{\prime} \subseteq \Gamma$ be a Lie subgroupoid integrating the Lie subalgebroid $\varphi\left(T^{*} Q\right)$. For any $x \in Q$, we have $T_{x} \Gamma=T_{x} P \oplus T_{x}^{\alpha} \Gamma$ and $T_{x} \Gamma^{\prime}=T_{x} Q \oplus T_{x}^{\alpha} \Gamma^{\prime}$. By identifying $T_{x}^{\alpha} \Gamma$ with $T_{x}^{*} P$ via $\omega^{b}$ as above, one obtains a decomposition $T_{x} \Gamma \cong T_{x} P \oplus T_{x}^{*} P$, under which the symplectic form $\omega_{x} \in \wedge^{2} T_{x}^{*} \Gamma$ takes the form:

$$
\left(\begin{array}{cc}
0 & I \\
-I & \pi(x)
\end{array}\right)
$$

Now $T_{x} P=T_{x} Q \oplus V_{x}$ and $T_{x}^{*} P=T_{x} Q^{\perp} \oplus V_{x}^{\perp} \cong V_{x}^{*} \oplus T_{x}^{*} Q$. Thus

$$
T_{x} \Gamma \cong T_{x} Q \oplus V_{x} \oplus V_{x}^{*} \oplus T_{x}^{*} Q .
$$


It is clear that under this decomposition $T_{x} \Gamma^{\prime}$ corresponds to the subspace $T_{x} Q \oplus T_{x}^{*} Q$. Thus the restriction of $\omega_{x}$ to the subspace $T_{x} \Gamma^{\prime}$ has the form:

$$
\left(\begin{array}{cc}
0 & I \\
-I & \pi_{Q}(x)
\end{array}\right)
$$

which is clearly non-degenerate. It follows immediately that the pull back of the symplectic form $\omega$ is non-degenerate along the identity section $Q$. To show its non-degeneracy at every point of $\Gamma^{\prime}$, it suffices to show that through each point of $\Gamma^{\prime}$, there exists a Lagrangian (local) bisection $S$ of $\Gamma$ such that $\left.S\right|_{Q}$ is a bisection of $\Gamma^{\prime}$. This is true since any closed one-form on $Q$ extends locally to a closed one-form on $P$.

\section{Poisson involutions}

This section is devoted to the study on a special class of Dirac submanifolds arising as the stable locus of a Poisson involution. In particular, we discuss Poisson involutions on Poisson groupoids as well as on Poisson groups. As we will see, such involutions often exist. Examples include the standard Poisson group structures on semi-simple Lie groups, Bruhat Poisson structures on compact semi-simple Lie groups, and Poisson groupoids associated with dynamical $r$-matrices of semi-simple Lie algebras.

\subsection{Stable locus of a Poisson involution}

Recall that a Poisson involution on a Poisson manifold $P$ is a Poisson diffeomorphism $\Phi: P \rightarrow P$ such that $\Phi^{2}=i d$. An important class of Dirac manifolds arises as follows.

Proposition 4.1. - Let $\Phi: P \rightarrow P$ be a Poisson involution. Then its stable locus $Q$ is a Dirac submanifold.

Proof. - It is well known that $Q$ is a smooth manifold. For any $x \in Q$, since the linear morphism $\Phi_{*}: T_{x} P \rightarrow T_{x} P$ is an involution, its eigenvalues are either +1 or -1 . Let $V_{x}$ denote the (-1)-eigenspace of $\Phi_{*}$, and $V_{Q}=\bigcup_{x \in Q} V_{x}$. Clearly $T_{x} Q$ coincides with the (+1)-eigenspace of $\Phi_{*}$, and $T_{x} P=T_{x} Q \oplus V_{x}$. Since $\Phi_{*} \pi=\pi$, it is clear that $\left.\pi\right|_{Q}=\pi_{Q}+\pi^{\prime}$, where $\pi_{Q} \in \Gamma\left(\wedge^{2} T Q\right)$ and $\pi^{\prime} \in \Gamma\left(\wedge^{2} V_{Q}\right)$. It remains to verify the condition (ii) of Proposition 2.8. For this purpose, note that any vector field $X$ on $P$ can be decomposed as $X=X^{+}+X^{-}$, where $\Phi_{*} X^{+}=X^{+}$ and $\Phi_{*} X^{-}=-X^{-}$. Indeed,

$$
X^{+}=\frac{1}{2}\left(X+\Phi_{*} X\right) \quad \text { and } \quad X^{-}=\frac{1}{2}\left(X-\Phi_{*} X\right) .
$$

It thus suffices to prove that $\operatorname{pr}_{*}\left[X^{-}, \pi\right]=0$. This is obvious since

$$
\Phi_{*}\left[X^{-}, \pi\right]=\left[\Phi_{*} X^{-}, \Phi_{*} \pi\right]=-\left[X^{-}, \pi\right] .
$$

As a consequence, the stable locus of a Poisson involution carries a natural Poisson structure. This observation was hidden in the work of Bondal [4] and Boalch [2] in their study of the Poisson structures on the space of Stokes matrices. On the other hand, an algebraic version of this fact recently appeared in the work of Fernandes and Vanhaecke [17]. Below we give an explicit description of such a Poisson tensor. 
Proposition 4.2. - Let $Q$ be the stable locus of a Poisson involution $\Phi: P \rightarrow P$. Assume that the Poisson tensor $\pi$ on $P$ is $\pi=\sum_{i} X_{i} \wedge Y_{i}$, where $X_{i}$ and $Y_{i}$ are vector fields on $P$. Then the Poisson tensor $\pi_{Q}$ on $Q$ is given by $\pi_{Q}=\left.\sum_{i} X_{i}^{+} \wedge Y_{i}^{+}\right|_{Q}$, where $X_{i}^{+}$and $Y_{i}^{+}$are defined by Eq. (15).

As a consequence of Theorem 3.8, we have the following

Corollary 4.3. - If $Q$ is the stable locus of a Poisson involution on an integrable Poisson manifold $P$, then $Q$ is always an integrable Poisson manifold itself.

Proof. - Assume that $Q$ is the stable locus of a Poisson involution $\Phi: P \rightarrow P$. Let $\Gamma$ be an $\alpha$ connected and simply connected symplectic groupoid of $P$. To the Poisson involution $\Phi: P \rightarrow P$, there corresponds an involutive symplectic groupoid automorphism $\widetilde{\Phi}: \Gamma \rightarrow \Gamma$. Then the stable locus of $\widetilde{\Phi}$, which is a smooth manifold, is a symplectic subgroupoid of $\Gamma$ integrating $Q$.

\subsection{Poisson involutions on Poisson groupoids}

For Poisson groupoids, there is an effective way of producing Poisson involutions. This is via the so called symmetric Poisson groupoids. A special case of these, namely, symmetric Poisson groups and their infinitesimal version, symmetric Lie bialgebras, were studied by Fernandes [14, 15]. ${ }^{2}$

\section{DEFINITION 4.4. -}

(i) A symmetric Poisson groupoid consists of a pair $(\Gamma, \Phi)$, where $\Gamma$ is a Poisson groupoid and $\Phi: \Gamma \rightarrow \Gamma$ is a groupoid anti-morphism which is also a Poisson involution.

(ii) A symmetric Lie bialgebroid consists of a triple $\left(A, A^{*}, \varphi\right)$, where $\left(A, A^{*}\right)$ is a Lie bialgebroid and $\varphi: A \rightarrow A$ is an involutive Lie algebroid anti-morphism such that $\varphi^{*}: A^{*} \rightarrow A^{*}$ is a Lie algebroid morphism.

THEOREM 4.5. - Under the assumption that the relevant Lie algebroid be integrable, there is a one-to-one correspondence between $\alpha$-simply connected symmetric Poisson groupoids and symmetric Lie bialgebroids.

Proof. - Assume that $\left(A, A^{*}, \varphi\right)$ is a symmetric Lie bialgebroid. Let $\Gamma$ be an $\alpha$-simply connected Poisson groupoid corresponding to the Lie bialgebroid $\left(A, A^{*}\right)$. It is known that any Lie algebroid isomorphism integrates to a Lie groupoid isomorphism for $\alpha$-simply connected Lie groupoids. Hence the Lie algebroid involution $\varphi^{\prime}=-\varphi: A \rightarrow A$ integrates to a Lie groupoid involution $\Phi^{\prime}: \Gamma \rightarrow \Gamma$. By assumption, $\left(\varphi^{\prime}\right)^{*}=(-\varphi)^{*}=-\varphi^{*}$ is a Lie algebroid anti-morphism. By the Poisson groupoid duality [27,28], $\Phi^{\prime}$ is an anti-Poisson map. Let $\tau: \Gamma \rightarrow \Gamma$ be the groupoid inversion: $\tau(g)=g^{-1}, \forall g \in \Gamma$, which is clearly a groupoid anti-morphism and an anti-Poisson map. Set $\Phi=\Phi^{\prime} \circ \tau$. Then $\Phi$ is an integration of $\varphi$, which possesses all the required properties.

Conversely, if $(\Gamma, \Phi)$ is a symmetric Poisson groupoid, it is clear that $\left(A, A^{*}, \varphi\right)$ is a symmetric Lie bialgebroid, where $\varphi: A \rightarrow A$ is the derivative of $\Phi$.

Remark 4.6. - Note that the roles of $A$ and $A^{*}$ can be switched for a symmetric Lie bialgebroid. Namely, if $\left(A, A^{*}, \varphi\right)$ is a symmetric Lie bialgebroid, then $\left(A^{*}, A,-\varphi^{*}\right)$ is also a symmetric Lie bialgebroid. This means that from a symmetric Lie bialgebroid one can in fact construct a pair of Poisson involutions: one on $\Gamma$ and the other on its dual Poisson groupoid $\Gamma^{*}$ (provided that both $A$ and $A^{*}$ are integrable).

\footnotetext{
${ }^{2}$ However note that our definition here is the opposite to that in $[14,15]$. We require that $\Phi$ be a group(oid) antimorphism and a Poisson map, while in [14,15] $\Phi$ is required to be a group morphism and an anti-Poisson map. To get from one notion to the other, one needs to compose $\Phi$ with the group(oid) inversion.
} 
Theorem 4.5 indicates that a useful source of producing Poisson involutions on Poisson groupoids is to construct symmetric Lie bialgebroids. Next we will consider the case of coboundary Lie bialgebroids [22], namely those Lie bialgebroids $\left(A, A^{*}\right)$ where the Lie algebroid structure on the dual $A^{*}$ is generated by an $r$-matrix $\Lambda \in \Gamma\left(\wedge^{2} A\right)$ with the property $[X,[\Lambda, \Lambda]]=0, \forall X \in \Gamma(A)$.

Proposition 4.7. - A coboundary Lie bialgebroid $\left(A, A^{*}\right)$ with an $r$-matrix $\Lambda \in \Gamma\left(\wedge^{2} A\right)$ is a symmetric Lie bialgebroid if there is an involutive Lie algebroid anti-morphism $\varphi: A \rightarrow A$ such that $\varphi \Lambda=-\Lambda$.

Proof. - Let $d_{*}: \Gamma\left(\wedge^{\bullet} A\right) \rightarrow \Gamma\left(\wedge^{\bullet+1} A\right)$ be the exterior differential induced from the Lie algebroid structure on $A^{*}$. Then for any $X \in \Gamma\left(\wedge^{\bullet} A\right), d_{*} X=[\Lambda, X]$. Hence assuming that $\varphi \Lambda=-\Lambda,\left(\varphi \circ d_{*}\right) X=\varphi[\Lambda, X]=-[\varphi \Lambda, \varphi X]=[\Lambda, \varphi X]=\left(d_{*} \circ \varphi\right) X$, which implies that $\varphi \circ d_{*}=d_{*} \circ$. Therefore $\varphi^{*}: A^{*} \rightarrow A^{*}$ is a Lie algebroid morphism.

\subsection{Symmetric Courant algebroids}

A nice way of understanding a Lie bialgebroid $\left(A, A^{*}\right)$ is via its double $E=A \oplus A^{*}$, which is a Courant algebroid [20]. Roughly speaking, a Courant algebroid is a vector bundle $E \rightarrow M$ equipped with a non-degenerate symmetric bilinear form $(\cdot, \cdot)$ of signature $(n, n)$ on the fibers, a bundle map $\rho: E \rightarrow T M$, and a bracket $[\cdot, \cdot]$ on $\Gamma(E)$, which satisfy compatibility conditions resembling those of a Lie algebroid up to a homotopy. Lie bialgebroids correspond to splittable Courant algebroids, namely those which admit two transversal Dirac structures. We refer the reader to [20] for details.

DEFINITION 4.8.-

(i) A symmetric Courant algebroid is a Courant algebroid $(E,(\cdot, \cdot), \rho,[\cdot, \cdot])$ equipped with an involutive anti-morphism $\chi: E \rightarrow E$, i.e.,

$$
\rho \circ \chi=-f_{* \circ \rho} ; \quad\left(\chi e_{1}, \chi e_{2}\right)=-\left(e_{1}, e_{2}\right) ; \quad \text { and } \quad \chi\left[e_{1}, e_{2}\right]=-\left[\chi e_{1}, \chi e_{2}\right]
$$

for any $e_{1}, e_{2} \in \Gamma(E)$, where $f: M \rightarrow M$ is the base map of $\chi$;

(ii) A symmetric splittable Courant algebroid is a symmetric Courant algebroid $(E, \chi)$, such that $E$ admits a pair of $\chi$-stable transversal Dirac structures.

THEOREM 4.9. - There is a one-to-one correspondence between symmetric Lie bialgebroids and symmetric splittable Courant algebroids.

Proof. - Assume that $\left(A, A^{*}, \varphi\right)$ is a symmetric Lie bialgebroid. Let $M$ denote the base of the Lie bialgebroid $\left(A, A^{*}\right)$, and $a, a_{*}$ the anchors of $A$ and $A^{*}$ respectively. Denote, by $f: M \rightarrow M$, the involution on the base manifold corresponding to $\varphi$. Then $\varphi^{*}$ is a bundle map over the same base map $f: M \rightarrow M$ since $f$ is an involution. Let $E=A \oplus A^{*}$ denote the double of the Lie bialgebroid, which is a Courant algebroid [20] over the base manifold $M$, with anchor $\rho=a+a_{*}$. Define $\chi: E \rightarrow E$ by

$$
\chi(X+\xi)=\varphi X-\varphi^{*} \xi,\left.\quad \forall X \in A\right|_{m} \text { and }\left.\xi \in A^{*}\right|_{m} .
$$

Clearly $\chi$ is an involutive bundle map over the base map $f: M \rightarrow M$. It is also simple to check that $\chi$ anti-commutes with the anchor on $E$, and $\left(\chi e_{1}, \chi e_{2}\right)=-\left(e_{1}, e_{2}\right)$ for any $e_{1}, e_{2} \in \Gamma(E)$. It remains to check that $\left[\chi e_{1}, \chi e_{2}\right]=-\left[e_{1}, e_{2}\right]$ for any $e_{1}, e_{2} \in \Gamma(E)$. To this end, it suffices to show that $[\chi X, \chi \xi]=-[X, \xi]$ for any $X \in \Gamma(A)$ and $\xi \in \Gamma\left(A^{*}\right)$. First we will need the following identities: 


$$
\begin{aligned}
& L_{\varphi^{*} \xi} \varphi X=\varphi\left(L_{\xi} X\right) ; \\
& L_{\varphi X} \varphi^{*} \xi=-\varphi^{*}\left(L_{X} \xi\right) .
\end{aligned}
$$

Note that for any $\eta \in \Gamma\left(A^{*}\right)$,

$$
\begin{aligned}
\left\langle L_{\varphi^{*} \xi} \varphi X, \eta\right\rangle & =\left(a_{*} \varphi^{*} \xi\right)\langle\varphi X, \eta\rangle-\left\langle\varphi X,\left[\varphi^{*} \xi, \eta\right]\right\rangle \\
& =f_{*}\left(a_{*} \xi\right)\langle\varphi X, \eta\rangle-\left\langle\varphi X,\left[\varphi^{*} \xi, \eta\right]\right\rangle \\
& =\left(a_{*} \xi\right)\left\langle X, \varphi^{*} \eta\right\rangle-\left\langle X, \varphi^{*}\left[\varphi^{*} \xi, \eta\right]\right\rangle \\
& =\left(a_{*} \xi\right)\left\langle X, \varphi^{*} \eta\right\rangle-\left\langle X,\left[\xi, \varphi^{*} \eta\right]\right\rangle \\
& =\left\langle L_{\xi} X, \varphi^{*} \eta\right\rangle \\
& =\left\langle\varphi\left(L_{\xi} X\right), \eta\right\rangle .
\end{aligned}
$$

Eq. (17) thus follows. Eq. (18) can be proved similarly. Now

$$
\begin{aligned}
{[\chi X, \chi \xi] } & =-\left[\varphi X, \varphi^{*} \xi\right] \\
& =L_{\varphi^{*} \xi} \varphi X-\frac{1}{2} d_{*}\left\langle\varphi^{*} \xi, \varphi X\right\rangle-L_{\varphi X} \varphi^{*} \xi+\frac{1}{2} d\left\langle\varphi^{*} \xi, \varphi X\right\rangle \quad \text { (by Eqs. (17)-(18)) } \\
& =\varphi\left(L_{\xi} X\right)-\frac{1}{2} \varphi d_{*}\langle\xi, X\rangle+\varphi^{*}\left(L_{X} \xi\right)-\frac{1}{2} \varphi^{*} d\langle\xi, X\rangle .
\end{aligned}
$$

On the other hand,

$$
\begin{aligned}
\chi[X, \xi] & =\chi\left[\left(-L_{\xi} X+\frac{1}{2} d_{*}\langle\xi, X\rangle\right)+\left(L_{X} \xi-\frac{1}{2} d\langle\xi, X\rangle\right)\right] \\
& =-\varphi\left(L_{\xi} X\right)+\frac{1}{2} \varphi d_{*}\langle\xi, X\rangle-\varphi^{*}\left(L_{X} \xi\right)+\frac{1}{2} \varphi^{*} d\langle\xi, X\rangle .
\end{aligned}
$$

Thus $[\chi X, \chi \xi]=-\chi[X, \xi]$.

Conversely, assume that $E$ is a splittable Courant algebroid such that $E=A \oplus A^{*}$ for a Lie bialgebroid $\left(A, A^{*}\right)$, and $\chi: E \rightarrow E$ is an involutive anti-morphism preserving both components $A$ and $A^{*}$. Let $\varphi=\left.\chi\right|_{A}: A \rightarrow A$ and $\psi=\left.\chi\right|_{A^{*}}: A^{*} \rightarrow A^{*}$. Then both $\varphi$ and $\psi$ are involutive Lie algebroid anti-morphisms. For any $X \in \Gamma(A)$ and $\xi \in \Gamma\left(A^{*}\right)$, since $(\chi \xi, \chi X)=-(\xi, X)$, and $\chi X=\varphi X, \chi \xi=\psi \xi$, it follows immediately that $\varphi^{*} \psi=-i d$, which implies that $\psi=-\varphi^{*}$. This concludes the proof of the theorem.

\subsection{Poisson involutions on dynamical Poisson groupoids}

As a special case, we will consider the dynamical Poisson groupoids introduced by Etingof and Varchenko [12]. Recall that a dynamical $r$-matrix on a Lie algebra $\mathfrak{g}$ with Lie subalgebra $\mathfrak{h}$ is a function $r: \mathfrak{h}^{*} \rightarrow \wedge^{2} \mathfrak{g}$ satisfying:

(i) $r: \mathfrak{h}^{*} \rightarrow \wedge^{2} \mathfrak{g}$ is $H$-equivariant;

(ii) $\sum_{i} h_{i} \wedge \frac{\partial r}{\partial \lambda^{2}}-\frac{1}{2}[r, r]$ is a constant $\left(\wedge^{2} \mathfrak{g}\right)^{\mathfrak{g}}$-valued function on $\mathfrak{h}^{*}$,

where $H$ is the connected Lie subgroup of $G$ with Lie algebra $\mathfrak{h},\left\{h_{1}, \ldots, h_{k}\right\}$ is a basis of $\mathfrak{h}$, and $\left\{\lambda_{1}, \ldots, \lambda_{k}\right\}$ are the induced coordinates on $\mathfrak{h}^{*}$.

It is known [1,23] that a dynamical $r$-matrix naturally defines a coboundary Lie bialgebroid $\left(A, A^{*}, \Lambda\right)$, where $A=T \mathfrak{h}^{*} \times \mathfrak{g}$, and $\Lambda=\pi_{\mathfrak{h}^{*}}+\sum_{i=1}^{k}\left(\frac{\partial}{\partial \lambda_{i}} \wedge h_{i}\right)+r(\lambda) \in \Gamma\left(\wedge^{2} A\right)$. Here $\pi_{\mathfrak{h}^{*}}$ is the Lie-Poisson tensor on $\mathfrak{h}^{*}$.

The following theorem can be verified directly. 
THEOREM 4.10. - Let $r: \mathfrak{h}^{*} \rightarrow \wedge^{2} \mathfrak{g}$ be a dynamical $r$-matrix. Assume that $s: \mathfrak{g} \rightarrow \mathfrak{g}$ is an involutive Lie algebra anti-morphism, which preserves $\mathfrak{h}$ and satisfies the property

$$
s(r(\lambda))=-r\left(s_{\mathfrak{h}}^{*} \lambda\right)
$$

$\forall \lambda \in \mathfrak{h}^{*}$. Here $s_{\mathfrak{h}}: \mathfrak{h} \rightarrow \mathfrak{h}$ is the restriction of $s$ to $\mathfrak{h}$. Then $\left(T \mathfrak{h}^{*} \times \mathfrak{g}, T^{*} \mathfrak{h}^{*} \times \mathfrak{g}^{*}, \varphi\right)$, where $\varphi=\left(-T s_{\mathfrak{h}}^{*}, s\right): T \mathfrak{h}^{*} \times \mathfrak{g} \rightarrow T \mathfrak{h}^{*} \times \mathfrak{g}$, is a symmetric Lie bialgebroid.

Corollary 4.11. - Under the same hypothesis as in Theorem 4.10, let $S: G \rightarrow G$ be the group anti-morphism corresponding to $s$. Then,

(i) $\Phi: \mathfrak{h}^{*} \times \mathfrak{h}^{*} \times G \rightarrow \mathfrak{h}^{*} \times \mathfrak{h}^{*} \times G, \Phi(u, v, g)=\left(s_{\mathfrak{h}}^{*} v, s_{\mathfrak{h}}^{*} u, S(g)\right)$, $\forall u, v \in \mathfrak{h}^{*}$ and $g \in G$, is a Poisson involution of the dynamical Poisson groupoid $\mathfrak{h}^{*} \times \mathfrak{h}^{*} \times G$.

(ii) $\Gamma^{0}=\left\{\left(u, s_{\mathfrak{h}}^{*} u, g\right) \mid \forall u \in \mathfrak{h}^{*}, g \in G^{0}\right\}$ is a Dirac submanifold, where $G^{0} \subset G$ is the stable locus of $S$.

Example 4.12. - Let $\mathfrak{g}$ be a semi-simple Lie algebra over $\mathbb{C}$ of rank $k$ with a Cartan subalgebra $\mathfrak{h}$. Let $\left\{e_{\alpha}, f_{\alpha}, h_{i} \mid \alpha \in \Delta_{+}, 1 \leqslant i \leqslant k\right\}$ be a Chevalley basis. Then

$$
r(\lambda)=\sum_{\alpha \in \Delta_{+}} d_{\alpha} \operatorname{coth}\left(\frac{1}{2}\langle\alpha, \lambda\rangle\right) e_{\alpha} \wedge f_{\alpha}
$$

is a dynamical $r$-matrix over $\mathfrak{h}^{*}$, where $\left(e_{\alpha}, f_{\alpha}\right)=d_{\alpha}$, and $\operatorname{coth}(x)=\frac{e^{x}+e^{-x}}{e^{x}-e^{-x}}$ is the hyperbolic cotangent function [12].

Let $s: \mathfrak{g} \rightarrow \mathfrak{g}$ be a $\mathbb{C}$-linear morphism, which, on generators, is defined as follows: ${ }^{3}$

$$
s e_{\alpha}=f_{\alpha}, \quad s f_{\alpha}=e_{\alpha}, \quad s h_{i}=h_{i} .
$$

It is clear that $s$ is an involutive Lie algebra anti-morphism and $\left.s\right|_{\mathfrak{h}}=i d$. Moreover, it is also clear that $s(r(\lambda))=-r(\lambda)$ for any $\lambda \in \mathfrak{h}^{*}$. Therefore, according to Theorem 4.10, $\left(T \mathfrak{h}^{*} \times \mathfrak{g}, T^{*} \mathfrak{h}^{*} \times \mathfrak{g}^{*}, \varphi\right)$ is a symmetric Lie bialgebroid, where $\varphi: T \mathfrak{h}^{*} \times \mathfrak{g} \rightarrow T \mathfrak{h}^{*} \times \mathfrak{g}$ is given by $\varphi(v, X)=(-v, s X), \forall(v, X) \in T \mathfrak{h}^{*} \times \mathfrak{g}$. Thus one obtains a pair of Poisson involutions on the corresponding Poisson groupoids $\Phi: \Gamma \rightarrow \Gamma$ and $\Psi: \Gamma^{*} \rightarrow \Gamma^{*}$. Now $\Gamma=\mathfrak{h}^{*} \times \mathfrak{h}^{*} \times G$, and $\Phi(u, v, g)=(v, u, S g), \forall u, v \in \mathfrak{h}^{*}$ and $g \in G$. Hence, the stable locus of $S$ is diffeomorphic to $\mathfrak{h}^{*} \times G^{0}$, where $G^{0}$ is the stable locus of $S$. It would be interesting to compute explicitly the induced Poisson structure on $\mathfrak{h}^{*} \times G^{0}$. On the other hand, it is not obvious what the stable locus of $\Psi$ should look like (see [19] for the description of the dual Poisson groupoid $\Gamma^{*}$ ).

Let $\mathfrak{l}$ be a reductive Lie subalgebra of $\mathfrak{g}$ containing $\mathfrak{h}$, i.e.,

$$
\mathfrak{l}=\mathfrak{h} \oplus \bigoplus_{\alpha \in \Delta_{+}^{\prime}}\left(\mathfrak{g}_{\alpha} \oplus \mathfrak{g}_{-\alpha}\right)
$$

where $\Delta_{+}^{\prime}$ is some subset of $\Delta_{+}$.

We now show that the claim in Example 4.12 in fact holds in the more general situation where $\mathfrak{h}$ is replaced by $\mathfrak{l}$.

PROPOSITION 4.13. - Let $\mathfrak{l}$ be a reductive Lie subalgebra of a semi-simple Lie algebra $\mathfrak{g}$ as in Eq. (20), and $r: \mathfrak{l}^{*} \rightarrow \wedge^{2} \mathfrak{g}$ a dynamical $r$-matrix. Then the map $s: \mathfrak{g} \rightarrow \mathfrak{g}$ defined by Eq. (19)

\footnotetext{
${ }^{3}$ Note that $-s$ is the Cartan involution of the split real form.
} 
satisfies the conditions of Theorem 4.10 , and therefore $\left(T \mathfrak{l}^{*} \times \mathfrak{g}, T^{*} \mathfrak{l}^{*} \times \mathfrak{g}^{*}, \varphi\right)$ is a symmetric Lie bialgebroid. Here $\varphi=\left(-T s_{\mathfrak{l}}^{*}, s\right): T \mathfrak{l}^{*} \times \mathfrak{g} \rightarrow T \mathfrak{l}^{*} \times \mathfrak{g}$.

Proof. - We prove this proposition using the classification result in [12]. Let $r_{0}: \mathfrak{h}^{*} \rightarrow \wedge^{2} \mathfrak{g}$ be the function:

$$
r_{0}(\lambda)=\sum_{\alpha \in \Delta_{+}^{\prime}} \frac{1}{(\alpha, \lambda)} e_{\alpha} \wedge f_{\alpha} .
$$

According to [12], $\tilde{r}=\left.r\right|_{\mathfrak{h}^{*}}+r_{0}: \mathfrak{h}^{*} \rightarrow \wedge^{2} \mathfrak{g}$ is a classical dynamical $r$-matrix on $\mathfrak{h}^{*}$. Hence from Example 4.12 (the rational case can also be similarly checked), we know that $s(\tilde{r}(\lambda))=-\tilde{r}(\lambda), \forall \lambda \in \mathfrak{h}^{*}$, which in turn implies that $s(r(\lambda))=-r(\lambda), \forall \lambda \in \mathfrak{h}^{*}$.

Now assume that $\mu=A d_{x^{-1}}^{*} \lambda \in \mathfrak{l}^{*}$, where $\lambda \in \mathfrak{h}^{*}$ and $x \in L$. By $S: G \rightarrow G$, we denote the group anti-morphism corresponding to $s$. Then

$$
\begin{aligned}
s(r(\mu)) & \left.=s\left(r\left(A d_{x^{-1}}^{*} \lambda\right)\right) \quad \text { (since } r \text { is } L \text {-equivariant }\right) \\
& =s\left[A d_{x} r(\lambda)\right]=A d_{S x^{-1}} s(r(\lambda)) \\
& =A d_{S x^{-1}}(-r(\lambda))=-r\left(A d_{S x}^{*} \lambda\right) \\
& =-r\left(s^{*} A d_{x^{-1}}^{*} s^{*} \lambda\right)=-r\left(s^{*} A d_{x^{-1}}^{*} \lambda\right) \\
& =-r\left(s^{*} \mu\right) .
\end{aligned}
$$

Here we used the identities: $s \circ A d_{x}=A d_{S x^{-1} \circ s}$ and $A d_{S x}^{*}=s^{*} \circ A d_{x^{-1} \circ s^{*}}^{*}$. Since those points $\mu=A d_{x^{-1}} \lambda, \forall \lambda \in \mathfrak{h}^{*}, x \in L$, consist of a dense subset of $\mathfrak{l}^{*}$, the conclusion follows immediately.

\section{Poisson involutions on Poisson groups}

In this section we turn our attention to Poisson involutions on Poisson groups.

\subsection{Symmetric Poisson groups}

As a special case of Definition 4.4, we have

DEFINITION 5.1.-

(i) A symmetric Poisson group consists of a pair $(G, \Phi)$, where $G$ is a Poisson group, and $\Phi: G \rightarrow G$ is a group anti-morphism which is also a Poisson involution.

(ii) A symmetric Lie bialgebra consists of a triple $\left(\mathfrak{g}, \mathfrak{g}^{*}, \varphi\right)$, where $\left(\mathfrak{g}, \mathfrak{g}^{*}\right)$ is a Lie bialgebra and $\varphi: \mathfrak{g} \rightarrow \mathfrak{g}$ is an involutive Lie algebra anti-morphism such that $\varphi^{*}: \mathfrak{g}^{*} \rightarrow \mathfrak{g}^{*}$ is a Lie algebra morphism.

In this case, a combination of Theorems 4.5 and 4.9 leads to the following:

THEOREM 5.2.-

(i) There is a one-to-one correspondence between simply connected symmetric Poisson groups and symmetric Lie bialgebras.

(ii) There is a one-to-one correspondence between symmetric Lie bialgebras $\left(\mathfrak{g}, \mathfrak{g}^{*}, \varphi\right)$ and involutive anti-morphisms $\chi: \sigma \rightarrow \sigma$ (i.e., $\left(\chi e_{1}, \chi e_{2}\right)=-\left(e_{1}, e_{2}\right)$; and $\left.\chi\left[e_{1}, e_{2}\right]=-\left[\chi e_{1}, \chi e_{2}\right]\right)$ of the double $\sigma=\mathfrak{g} \oplus \mathfrak{g}^{*}$ preserving both components $\mathfrak{g}$ and $\mathfrak{g}^{*}$.

(iii) If $\left(\mathfrak{g}, \mathfrak{g}^{*}\right)$ is a coboundary Lie bialgebra with an r-matrix $r \in \wedge^{2} \mathfrak{g}$, then $\left(\mathfrak{g}, \mathfrak{g}^{*}, \varphi\right)$ is a symmetric Lie bialgebra if $\varphi: \mathfrak{g} \rightarrow \mathfrak{g}$ is an involutive Lie algebra anti-morphism such that $\varphi r=-r$. 
Now assume that $\left(\mathfrak{g}, \mathfrak{g}^{*}, \varphi\right)$ is a symmetric Lie bialgebra. According to the proof of Theorem 4.9, $\chi: \sigma \rightarrow \sigma, \chi(X+\xi)=\varphi X-\varphi^{*} \xi, \forall X+\xi \in \mathfrak{g} \oplus \mathfrak{g}^{*}$, is an involutive Lie algebra anti-morphism, where $\sigma=\mathfrak{g} \oplus \mathfrak{g}^{*}$ is the double of the Lie bialgebra. On the other hand, it is well known that $\left(\sigma, \sigma^{*}\right)$ itself is a Lie bialgebra with the $r$-matrix: $r=\sum_{i} X_{i} \wedge \xi^{i} \in \wedge^{2} \sigma$, where $\left\{X_{1}, \ldots, X_{n}\right\}$ is a basis of $\mathfrak{g}$ and $\left\{\xi^{1}, \ldots, \xi^{n}\right\}$ is the dual basis of $\mathfrak{g}^{*}$. Then

$$
\chi(r)=-\sum_{i} \varphi X_{i} \wedge \varphi^{*} \xi^{i}=-r
$$

since $\left\{\varphi^{*} \xi^{1}, \ldots, \varphi^{*} \xi^{n}\right\}$ is the basis dual to $\left\{\varphi X_{1}, \ldots, \varphi X_{n}\right\}$. Thus we have proved the following:

PROPOSITION 5.3. - The double of a symmetric Lie bialgebra is a symmetric Lie bialgebra.

Remark 5.4. - Let $D$ be a simply connected and connected Lie group with Lie algebra $\sigma$. The space $D$ possesses three different structures (under certain assumptions on completeness): a Poisson group, a symplectic groupoid $\Gamma_{G}$ over $G$ and a symplectic groupoid $\Gamma_{G^{*}}$ over $G^{*}$. If $\left(\mathfrak{g}, \mathfrak{g}^{*}, \varphi\right)$ is a symmetric Lie bialgebra, then $\varphi$ induces a Poisson involution on $D$, an involutive automorphism on the symplectic groupoid $\Gamma_{G}$, and an involutive automorphism on the symplectic groupoid $\Gamma_{G^{*}}$. These three involutions are different (see [4]). Their stable loci correspond to a Dirac submanifold of $D$, a symplectic groupoid over the stable locus of $\Phi$, and a symplectic groupoid over the stable locus of $\Psi$. Here $\Phi: G \rightarrow G$ and $\Psi: G^{*} \rightarrow G^{*}$ are the corresponding involutions induced by $\varphi$.

\subsection{Poisson structures on stable loci}

Below we outline a scheme to explicitly compute the Poisson tensor on the stable locus $Q$ of the Poisson involution $\Phi$ for a symmetric Poisson group $(G, \Phi)$. Since $\Phi$ is an involutive group anti-morphism, we have

$$
A d_{\Phi(x)^{-1} \circ} \varphi=\varphi_{\circ} A d_{x}: \mathfrak{g} \rightarrow \mathfrak{g}, \quad \forall x \in G .
$$

DEFINITION 5.5. - Let $\Phi: G \rightarrow G$ be an involutive group anti-morphism.

(i) A smooth map $\xi: G \rightarrow \wedge \cdot \mathfrak{g}$ is said to be $\Phi$-equivariant if

$$
\xi(\Phi(x))=A d_{\Phi(x)} \varphi(\xi(x)), \quad \forall x \in G
$$

(ii) It is said to be anti- $\Phi$-equivariant if

$$
\xi(\Phi(x))=-A d_{\Phi(x)} \varphi(\xi(x)), \quad \forall x \in G .
$$

Indeed, any smooth map $\xi: G \rightarrow \wedge^{\bullet} \mathfrak{g}$ can be decomposed as $\xi=\xi^{+}+\xi^{-}$such that $\xi^{+}$is $\Phi$-equivariant and $\xi^{-}$is anti- $\Phi$-equivariant, where

$$
\begin{aligned}
& \xi^{+}(x)=\frac{1}{2}\left[\xi(x)+\varphi\left(A d_{\Phi(x)^{-1}} \xi(\Phi(x))\right)\right] ; \\
& \xi^{-}(x)=\frac{1}{2}\left[\xi(x)-\varphi\left(A d_{\Phi(x)^{-1}} \xi(\Phi(x))\right)\right] .
\end{aligned}
$$

It is simple to see that $\xi: G \rightarrow \wedge^{\bullet} \mathfrak{g}$ is $\Phi$-equivariant (or anti- $\Phi$-equivariant) if and only if its right translation $r_{x *} \xi(x)$ is a $\Phi$-invariant (or anti- $\Phi$-invariant) multi-vector field on $G$. 
Let $\delta: \mathfrak{g} \rightarrow \wedge^{2} \mathfrak{g}$ denote the cobracket of the Lie bialgebra $\left(\mathfrak{g}, \mathfrak{g}^{*}\right)$, which is also a Lie algebra 1-cocycle, and let $\lambda: G \rightarrow \wedge^{2} \mathfrak{g}$ be its corresponding Lie group 1-cocycle. It is well known that $\pi(x)=r_{x *} \lambda(x), \forall x \in G$, is a Poisson tensor on the Poisson group $G$. Since $\pi$ is $\Phi$-invariant, it thus follows that $\lambda: G \rightarrow \wedge^{2} \mathfrak{g}$ is $\Phi$-equivariant.

Proposition 5.6. - Assume that the group 1-cocycle $\lambda: G \rightarrow \wedge^{2} \mathfrak{g}$ is $\lambda=\sum_{i} \xi_{i} \wedge \eta_{i}$, where $\xi_{i}, \eta_{i}: G \rightarrow \mathfrak{g}$. Then $\pi_{Q}(x)=\left.\sum_{i} r_{x *} \xi_{i}^{+}(x) \wedge r_{x *} \eta_{i}^{+}(x)\right|_{Q}$ is the Poisson tensor on the Dirac submanifold $Q$, where $\xi_{i}^{+}$and $\eta_{i}^{+}$are defined as in Eqs. (24)-(25). Moreover, the symplectic leaves of $Q$ are the intersection of $Q$ with the dressing orbits of $G^{*}$.

When $G$ is a coboundary Poisson group, one can write $\pi_{Q}$ more explicitly.

COROLlary 5.7.- In addition to the hypothesis of Theorem 5.2, assume that $G$ is a coboundary Poisson group with r-matrix $r=\sum_{i} e_{i} \wedge f_{i} \in \wedge^{2} \mathfrak{g}$. Then the Poisson tensor on $Q$ is given by

$$
\pi_{Q}=\left.\frac{1}{4} \sum_{i}\left(\overleftarrow{e_{i}}+\overrightarrow{\varphi e_{i}}\right) \wedge\left(\overleftarrow{f_{i}}+\overrightarrow{\varphi f_{i}}\right)\right|_{Q}-\left.\frac{1}{4} \sum_{i}\left(\overrightarrow{e_{i}}+\overleftarrow{\varphi e_{i}}\right) \wedge\left(\overrightarrow{f_{i}}+\overleftarrow{\varphi f_{i}}\right)\right|_{Q}
$$

where $\overleftarrow{e_{i}}$ and $\overrightarrow{e_{i}}$ are, respectively, the left- and right-invariant vector fields on G corresponding to $e_{i} \in \mathfrak{g}$; similarly for $\overleftarrow{f_{i}}$ and $\overrightarrow{f_{i}}$, etc.

In particular, if $e_{i}$ and $f_{i}$ are chosen such that $\varphi e_{i}=e_{i}$ and $\varphi f_{i}=-f_{i}$, then

$$
\pi_{Q}=\left.\frac{1}{2} \sum_{i}\left(\overleftarrow{e_{i}}+\overrightarrow{e_{i}}\right) \wedge\left(\overleftarrow{f_{i}}-\overrightarrow{f_{i}}\right)\right|_{Q}
$$

Proof. - It is simple to see, using Eq. (24), that for any $\xi \in \mathfrak{g}, \xi^{+}(x)=\frac{1}{2}\left(\xi+A d_{x}(\varphi \xi)\right)$ and $\left(A d_{x} \xi\right)^{+}(x)=\frac{1}{2}\left(A d_{x} \xi+\varphi \xi\right)$. It follows that

$$
r_{x *}\left(A d_{x} \xi\right)^{+}(x)=\frac{1}{2}(\overleftarrow{\xi}+\overrightarrow{\varphi \xi}) \quad \text { and } \quad r_{x *} \xi^{+}(x)=\frac{1}{2}(\vec{\xi}+\overleftarrow{\varphi \xi})
$$

It is well known that, for a coboundary Poisson group, $\lambda(x)=\sum_{i}\left(A d_{x} e_{i} \wedge A d_{x} f_{i}-e_{i} \wedge f_{i}\right)$. Eq. (26) thus follows immediately.

\subsection{Poisson symmetric spaces}

In what follows, we discuss the relation between the stable locus of the Poisson involution of a symmetric Poisson group and Poisson symmetric spaces. By a Poisson symmetric space for a given Poisson group $G$, we mean a symmetric $G$-space $P$ equipped with a Poisson structure such that the natural projection $G \rightarrow P$ is a Poisson map. In particular $P$ is a Poisson homogeneous space in the sense of Drinfel'd [10]. ${ }^{4}$

Assume that $(G, \Phi)$ is a symmetric Poisson group, and $Q=\{g \mid \Phi(g)=g\}$ is the stable locus of $\Phi$. The following result is standard (c.f. [31,32]). For completeness, we outline a proof below.

PROPOSITION 5.8. - Any connected component of $Q$ is a symmetric space.

Proof. - Let $g_{0} \in Q$ be any fixed point of $\Phi$, and $Q_{g_{0}}$ the connected component of $Q$ through $g_{0}$. Consider the twisted $G$-action on (the space) $G$ given by [31]:

$$
g \cdot x=g x \Phi(g), \quad \forall g, x \in G .
$$

\footnotetext{
${ }^{4}$ Note that our definition of Poisson symmetric spaces is different from that in $[14,15]$.
} 
Since $\Phi$ is a group anti-morphism, this is clearly an action. Now

$$
\Phi(g \cdot x)=\Phi(g x \Phi(g))=g \Phi(x) \Phi(g)=g \cdot \Phi(x),
$$

so $Q$ is stable under this action. Therefore in particular $Q_{g_{0}}$ is stable as well. Let $Q_{g_{0}}^{\prime}$ denote the $G$-orbit through $g_{0}$. Then $Q_{g_{0}}^{\prime}$ is a homogeneous space $Q_{g_{0}}^{\prime} \cong G / H_{g_{0}}$, where $H_{g_{0}}=\left\{g \mid g \in G, g g_{0} \Phi(g)=g_{0}\right\}$ is the isotropy group at $g_{0}$. Set

$$
\Phi_{g_{0}}: G \rightarrow G, \quad \Phi_{g_{0}}(g)=A d_{g_{0}} \Phi\left(g^{-1}\right), \quad \forall g \in G .
$$

Then $\Phi_{g_{0}}$ is an involutive group homomorphism, since

$$
\begin{aligned}
\Phi_{g_{0}}^{2}(g) & =\Phi_{g_{0}}\left(A d_{g_{0}} \Phi\left(g^{-1}\right)\right)=A d_{\Phi_{g_{0}}\left(g_{0}\right)} \Phi_{g_{0}}\left(\Phi\left(g^{-1}\right)\right) \\
& =A d_{g_{0}^{-1}} A d_{g_{0}} \Phi\left(\Phi\left(g^{-1}\right)\right)^{-1}=g, \quad \forall g \in G .
\end{aligned}
$$

It is clear that $H_{g_{0}}$ is the stable locus of $\Phi_{g_{0}}$. Hence $Q_{g_{0}}^{\prime}$ is indeed a symmetric space, and its dimension is equal to the dimension of the $(-1)$-eigenspace of $\varphi_{g_{0}}$, where $\varphi_{g_{0}}=-A d_{g_{0}} \circ \varphi: \mathfrak{g} \rightarrow \mathfrak{g}$ is the Lie algebra involution corresponding to $\Phi_{g_{0}}$. On the other hand, the tangent space $T_{g_{0}} Q_{g_{0}}$ is spanned by those vectors $v \in T_{g_{0}} G$ such that $\Phi_{*} v=v$. By identifying $T_{g_{0}} G$ with $\mathfrak{g}$ by right translation, $T_{g_{0}} Q_{g_{0}}$ can be identified with the subspace of $\mathfrak{g}$ consisting of those elements $X$ satisfying $A d_{g_{0}} \circ X=X$, i.e., the (-1)-eigenspace of $\varphi_{g_{0}}$. Therefore $Q_{g_{0}}^{\prime}$ is a submanifold of $Q_{g_{0}}$ of the same dimension, so it must be an open submanifold. Since it is also closed, they must be identical. This concludes the proof.

We are now ready to prove the following:

Theorem 5.9. - Let $(G, \Phi)$ be a symmetric Poisson group, and $Q=\{g \mid \Phi(g)=g\}$ the stable locus of $\Phi$. If the Poisson tensor $\pi$ on $G$ vanishes at a point $g_{0} \in Q$, then the connected component $Q_{g_{0}}$ is a Poisson symmetric space (scaled by a factor of 2). In particular, the identity component of $Q$ is a Poisson symmetric space.

Proof. - Consider the map

$$
f: G \rightarrow Q_{g_{0}}, \quad g \rightarrow g \cdot g_{0}=g g_{0} \Phi(g), \quad \forall g \in G .
$$

It suffices to prove that $f$ is a Poisson map, where $Q_{g_{0}}$ is equipped with the Poisson tensor $2 \pi_{Q}$. First, it is simple to see that

$$
f_{*} \delta_{g}=R_{g_{0} \Phi(g)} \delta_{g}+L_{g g_{0}} \Phi_{*} \delta_{g}, \quad \forall \delta_{g} \in T_{g} G .
$$

On the other hand, we have

$$
L_{g g_{0}} \Phi_{*} \delta_{g}=\Phi_{*}\left(R_{g_{0} \Phi(g)} \delta_{g}\right) .
$$

To see this, take a curve $g(t)$ starting at $g$ with $\left.\frac{d}{d t}\right|_{t=0} g(t)=\delta_{g}$. Since $\Phi$ is an involutive antimorphism, we have $g g_{0} \Phi(g(t))=\Phi\left(g(t) g_{0} \Phi(g)\right)$. Eq. (31) thus follows by taking the derivative at $t=0$. Combining Eq. (30) with Eq. (31), we are thus led to

$$
f_{*} \delta_{g}=2\left(R_{g_{0} \Phi(g)} \delta_{g}\right)^{+} .
$$

Now write $\pi(g)=\sum_{i j} \delta_{g}^{i} \wedge \delta_{g}^{j}$, where $\delta_{g}^{i}, \delta_{g}^{j} \in T_{g} G$. Then we have

$$
f_{*} \pi(g)=4 \sum_{i j}\left(R_{g_{0} \Phi(g)} \delta_{g}^{i}\right)^{+} \wedge\left(R_{g_{0} \Phi(g)} \delta_{g}^{j}\right)^{+} .
$$


On the other hand, from the multiplicativity condition of the Poisson tensor $\pi(g)$, it follows that

$$
\begin{aligned}
\pi\left(g g_{0} \Phi(g)\right) & =R_{g_{0} \Phi(g)} \pi(g)+L_{g} \pi\left(g_{0} \Phi(g)\right) \\
& =R_{g_{0} \Phi(g)} \pi(g)+L_{g g_{0}} \pi(\Phi(g)) \\
& =R_{g_{0} \Phi(g)} \pi(g)+L_{g g_{0}} \Phi_{*} \pi(g) \\
& =R_{g_{0} \Phi(g)} \pi(g)+\Phi_{*}\left(R_{g_{0} \Phi(g)} \pi(g)\right) \\
& =\sum R_{g_{0} \Phi(g)} \delta_{g}^{i} \wedge R_{g_{0} \Phi(g)} \delta_{g}^{j}+\sum \Phi_{*} R_{g_{0} \Phi(g)} \delta_{g}^{i} \wedge \Phi_{*} R_{g_{0} \Phi(g)} \delta_{g}^{j} .
\end{aligned}
$$

Here we used the assumption $\pi\left(g_{0}\right)=0$ in the second equality. Therefore we have

$$
\pi_{Q}\left(g g_{0} \Phi(g)\right)=2 \sum\left(R_{g_{0} \Phi(g)} \delta_{g}^{i}\right)^{+} \wedge\left(R_{g_{0} \Phi(g)} \delta_{g}^{j}\right)^{+} .
$$

This concludes the proof.

Remark 5.10.-

(i) Theorem 5.9 would follow from Theorem 3.7, if the action defined by Eq. (28) were a Poisson action where the Poisson group is equipped with the Poisson tensor $\pi(g)$ while the space upon which it acts, which is $G$ again, is equipped with $2 \pi(g)$. However, this is false in general. So we can see that a Poisson group action on a Poisson manifold $P$ may not be a Poisson action, but it can still be Poisson when restricted to the stable locus $Q$ of a Poisson involution.

(ii) One drawback of Theorem 5.9 is that the stable loci do not seem to produce any new examples of Poisson manifolds for symmetric Poisson groups in contradiction to what one may initially expect. A good point, on the other hand, is that one might be able to quantize these Poisson structures on stable loci including the one on Stokes matrices $U_{+}$ (see Example 5.11) using quantum homogeneous spaces.

(iii) One can construct a symplectic groupoid of a Poisson symmetric space by reduction [37]. On the other hand, according to Corollary 4.3, for a stable locus Poisson structure, one can construct a symplectic groupoid directly via the lifted involution on the corresponding symplectic groupoid. It would be interesting to compare these two approaches.

\subsection{Examples}

We end the paper with a list of examples. We refer the reader to [14] for a complete list of orthogonal symmetric Lie bialgeras, which also contains the examples below.

Example 5.11. - Let $\mathfrak{g}$ be a semi-simple Lie algebra of rank $k$ over $\mathbb{C}$ with a Cartan subalgebra $\mathfrak{h}$. Let $\left\{e_{\alpha}, f_{\alpha}, h_{i} \mid \alpha \in \Delta_{+}, 1 \leqslant i \leqslant k\right\}$ be a Chevalley basis. It is well known that $\left(\mathfrak{g}, \mathfrak{g}^{*}\right)$ is a coboundary Lie bialgebra with $r$-matrix:

$$
r=\sum_{\alpha \in \Delta_{+}} d_{\alpha}\left(e_{\alpha} \wedge f_{\alpha}\right)
$$

where $d_{\alpha}=\left(e_{\alpha}, f_{\alpha}\right)$.

As in Example 4.12, let $\varphi: \mathfrak{g} \rightarrow \mathfrak{g}$ be the $\mathbb{C}$-linear morphism, which, on generators, is defined as follows:

$$
\varphi e_{\alpha}=f_{\alpha}, \quad \varphi f_{\alpha}=e_{\alpha}, \quad \varphi h_{i}=h_{i}
$$


It is clear that $\varphi$ is an involutive Lie algebra anti-morphism and $\varphi r=-r$. Therefore $\left(\mathfrak{g}, \mathfrak{g}^{*}, \varphi\right)$ is a symmetric Lie bialgebra, which in turn induces a pair of symmetric Poisson groups $(G, \Phi)$ and $\left(G^{*}, \Psi\right)$. Thus one obtains a pair of Poisson involutions $\Phi: G \rightarrow G$ and $\Psi: G^{*} \rightarrow G^{*}$, which are the group anti-morphisms corresponding to the Lie algebra anti-morphisms $\varphi: \mathfrak{g} \rightarrow \mathfrak{g}$ and $-\varphi^{*}: \mathfrak{g}^{*} \rightarrow \mathfrak{g}^{*}$, respectively.

For $\mathfrak{g}=\mathfrak{s l}(n, \mathbb{C})$, it is well known that $G=S L(n, \mathbb{C})$ and

$$
G^{*}:=B_{+} * B_{-}=\left\{(B, C) \in B_{+} \times B_{-} \mid d(B) d(C)=1\right\},
$$

where $B_{+}$and $B_{-}$are the upper and lower triangular Borel subgroups of $G:=S L(n, \mathbb{C})$, and $d$ takes the diagonal part. It is simple to see that $\Phi$ and $\Psi$ are given by the following:

$$
\Phi: S L(n, \mathbb{C}) \rightarrow S L(n, \mathbb{C}), \quad \Phi(A)=A^{T}, \quad \forall A \in S L(n, \mathbb{C}) ;
$$

and

$$
\Psi: B_{+} * B_{-} \rightarrow B_{+} * B_{-}, \quad \Psi(B, C)=\left(C^{T}, B^{T}\right), \quad \forall(B, C) \in B_{+} * B_{-} .
$$

The stable locus of $\Phi$ thus consists of all symmetric matrices in $S L(n, \mathbb{C})$. On the other hand, the space $U_{+}$of Stokes matrices, i.e., upper triangular matrices with all main diagonal entries equal to 1 , can be identified with the identity component of the stable locus of $\Psi$. As a consequence, both the space $S$ of symmetric matrices in $S L(n, \mathbb{C})$ and the space $U_{+}$ of Stokes matrices admit natural Poisson structures. These Poisson manifolds, together with their symplectic groupoids, were studied in details by Bondal [4] in connection with his study of triangulated categories. The Poisson structure on $U_{+}$was also obtained independently by Dubrovin [11] in the $3 \times 3$-case and by Ugaglia [33] in the general $n \times n$-case in connection with the study of Frobenius manifolds. Boalch also realized, independently from Bondal, that this Poisson structure on $U_{+}$coincides with the induced Poisson structure on the stable locus of a Poisson involution on the Poisson group $B_{+} * B_{-}$[2]. We refer the reader to [2,4] for details.

As a consequence of Theorem 5.9, we conclude that both $S$ and $U_{+}$are indeed Poisson symmetric spaces.

THEOREM 5.12. - Scaled by a factor of 2, $S$ is a Poisson symmetric space for the Poisson group $G=S L(n, \mathbb{C})$, while $U_{+}$is a Poisson symmetric space for the dual Poisson group $G^{*}=B_{+} * B_{-}$. More precisely,

(i) the map $S L(n, \mathbb{C}) \rightarrow S, A \rightarrow A A^{T}, \forall A \in S L(n, \mathbb{C})$, is a Poisson map, and therefore $S$ is a Poisson symmetric space with the Poisson $S L(n, \mathbb{C})$-action:

$$
S L(n, \mathbb{C}) \times S \rightarrow S, \quad A \cdot X=A X A^{T}, \quad \forall A \in S L(n, \mathbb{C}), X \in S ;
$$

(ii) the map $B_{+} * B_{-} \rightarrow U_{+},(B, C) \rightarrow B C^{T}, \forall(B, C) \in B_{+} * B_{-}$is a Poisson map, and therefore $U_{+}$is a Poisson symmetric space with the Poisson $\left(B_{+} * B_{-}\right)$-action:

$$
\left(B_{+} * B_{-}\right) \times U_{+} \rightarrow U_{+}, \quad(B, C) \cdot X=B X C^{T}, \quad \forall(B, C) \in B_{+} * B_{-}, X \in U_{+} .
$$

Example 5.13. - Let $K$ be a compact semi-simple Lie group with Lie algebra $\mathfrak{k}$, and $\mathfrak{t}$ its Cartan subalgebra. It is well known that $K$ admits a standard Poisson group structure called Bruhat Poisson structure [25]. Let $\mathfrak{g}=\mathfrak{k}^{\mathbb{C}}$ be its complexification, which is a complex semisimple Lie algebra. Choose a Chevalley basis $\left\{e_{\alpha}, f_{\alpha}, h_{i} \mid \alpha \in \Delta_{+}, 1 \leqslant i \leqslant k\right\}$ of $\mathfrak{g}$ as in Example 5.11 such that $\left\{X_{\alpha}, Y_{\alpha}, t_{i} \mid \alpha \in \Delta_{+}, 1 \leqslant i \leqslant k\right\}$, where

$$
X_{\alpha}=e_{\alpha}-f_{\alpha}, \quad Y_{\alpha}=\sqrt{-1}\left(e_{\alpha}+f_{\alpha}\right), \quad \text { and } \quad t_{i}=\sqrt{-1} h_{i},
$$


is a basis (over $\mathbb{R}$ ) of $\mathfrak{k}$, and

$$
\hat{r}=\sqrt{-1} r=\sqrt{-1} \sum_{\alpha \in \Delta_{+}} d_{\alpha}\left(e_{\alpha} \wedge f_{\alpha}\right)=\sum_{\alpha \in \Delta_{+}} \frac{1}{2} d_{\alpha} X_{\alpha} \wedge Y_{\alpha} \in \wedge^{2} \mathfrak{k}
$$

is the $r$-matrix generating the corresponding Lie bialgebra $\left(\mathfrak{k}, \mathfrak{k}^{*}\right)$. Let $\varphi: \mathfrak{g} \rightarrow \mathfrak{g}$ be the antimorphism as in Example 4.12. It is then clear that $\varphi\left(X_{\alpha}\right)=-X_{\alpha}, \varphi\left(Y_{\alpha}\right)=Y_{\alpha}$, and $\varphi\left(t_{i}\right)=t_{i}$, so $\mathfrak{k}$ is stable under $\varphi$. It is also clear that $\varphi \hat{r}=-\hat{r}$. Hence $\left(\mathfrak{k}, \mathfrak{k}^{*}, \hat{\varphi}\right)$, where $\hat{\varphi}=\left.\varphi\right|_{\mathfrak{k}}: \mathfrak{k} \rightarrow \mathfrak{k}$, is a symmetric Lie bialgebra. Thus it induces a pair of Poisson involutions $\widehat{\Phi}: K \rightarrow K$ and $\widehat{\Psi}: K^{*} \rightarrow K^{*}$.

To describe the stable loci of these involutions, we need to consider the double of the Lie bialgebra $\left(\mathfrak{k}, \mathfrak{k}^{*}\right)$, which is isomorphic to $\mathfrak{g}$ as a real Lie algebra. According to Theorem 5.2, $\hat{\varphi}$ induces an involutive Lie algebra anti-morphism (over $\mathbb{R}$ ) $\chi: \mathfrak{g} \rightarrow \mathfrak{g}$, under which both $\mathfrak{k}$ and $\mathfrak{k}^{*}$ are stable and whose restrictions to these Lie subalgebras are $\hat{\varphi}$ and $-\hat{\varphi}^{*}$, respectively. In our case, a straightforward computation yields that on generators $\chi$ is given by:

$$
\begin{aligned}
& \chi\left(\sqrt{-1} e_{\alpha}\right)=\sqrt{-1} e_{\alpha}, \quad \chi\left(\sqrt{-1} f_{\alpha}\right)=\sqrt{-1} f_{\alpha}, \quad \chi\left(\sqrt{-1} h_{i}\right)=\sqrt{-1} h_{i}, \\
& \chi\left(e_{\alpha}\right)=-e_{\alpha}, \quad \chi\left(f_{\alpha}\right)=-f_{\alpha}, \quad \chi\left(h_{i}\right)=-h_{i} .
\end{aligned}
$$

In other words, $\chi=-\tau_{0}$, where $\tau_{0}$ is the complex conjugation on $\mathfrak{g}$ defined by the split real form spanned by $\left\{e_{\alpha}, f_{\alpha}, h_{i} \mid \alpha \in \Delta_{+}, 1 \leqslant i \leqslant k\right\}$. We will denote $\tau_{0}(X)=\bar{X}, \forall X \in \mathfrak{g}$. On the group level, $\chi$ induces an involutive Lie group anti-morphism $\Upsilon: G \rightarrow G$ such that $\Upsilon(g)=\bar{g}^{-1}$, $\forall g \in G$, where $G$ is a simply connected Lie group (considered as a real Lie group) integrating the Lie algebra $\mathfrak{g}$. By $Q$, we denote the stable locus of $\Upsilon$, i.e., $Q=\left\{g \in G \mid \bar{g}=g^{-1}\right\}$. Then the stable locus of $\widehat{\Phi}$ and $\widehat{\Psi}$ are $K \cap Q$ and $K^{*} \cap Q$, respectively. In particular, according to Corollary 5.7,

$$
\pi_{Q}=\sum_{\alpha \in \Delta_{+}} \frac{1}{4} d_{\alpha}\left(\overrightarrow{X_{\alpha}}-\overleftarrow{X_{\alpha}}\right) \wedge\left(\overleftarrow{Y_{\alpha}}+\overrightarrow{Y_{\alpha}}\right)
$$

is the Poisson tensor on $K \cap Q$. Theorem 5.9 implies that the map $g \rightarrow g \bar{g}^{-1}$ is indeed a Poisson map (scaled by a factor of 2) when being restricted to $K$ and $K^{*}$.

For $K=S U(n)$, its dual group $K^{*}$ is isomorphic to $S B(n, \mathbb{C})$, and the double $G \cong S L(n, \mathbb{C})$, considered as a real Lie group. Thus $Q=\{A \in S L(n, \mathbb{C}) \mid \bar{A} A=I\}$. Hence we have

$$
K \cap Q \cong\left\{A \mid A^{*} A=\bar{A} A=I, \operatorname{det} A=1\right\},
$$

which is the submanifold of $S U(n)$ consisting of all symmetric matrices. On the other hand,

$$
K^{*} \cap Q \cong\{A \in S B(n, \mathbb{C}) \mid \bar{A} A=I\} .
$$

We note that $S B(n, \mathbb{C})$ is Poisson diffeomorphic to the linear Poisson structure on $\mathfrak{s} \mathfrak{b}(n, \mathbb{C})$ according to Ginzburg-Weinstein theorem [18]. The recent result of Boalch [2] suggests that there may exist a Poisson diffeomorphism $S B(n, \mathbb{C}) \rightarrow \mathfrak{s b}(n, \mathbb{C})$ commuting with the Poisson involutions, where the Poisson involution on $S B(n, \mathbb{C})$ is given by $A \rightarrow \bar{A}^{-1}$ while the Poisson involution on $\mathfrak{s b}(n, \mathbb{C})$ is $A \rightarrow-\bar{A}$. If so, the induced Poisson structures on their stable locus should be isomorphic. The latter is a lot easier to compute and in fact is again a linear Poisson structure. 


\section{Acknowledgements}

The author would like to thank the Erwin Schrödinger Institute and the University of Pennsylvania for their hospitality while work on this project was being done. He also wishes to thank Philip Boalch, Alexei Bondal, Sam Evens, Rui Fernandes, Friedrich Knop, ZhangJu Liu, Jiang-Hua Lu and Alan Weinstein for numerous fruitful discussions, comments and suggestions. In particular, he is grateful to Alexei Bondal for allowing him to read his unpublished manuscripts $[3,4]$. He also thanks the referees for providing many suggestions in improving the presentation of the paper. Finally special thanks go to all his friends in Glanon, in particular, Frédéric Bidegain, Sébastien Michéa, and François Nadaud, from whom he benefited a lot not only mathematically, but also learned much more beyond.

\section{REFERENCES}

[1] Bangoura M., Kosmann-Schwarzbach Y., Équation de Yang-Baxter dynamique classique et algébroïdes de Lie, C. R. Acad. Sci. Paris, Série I 327 (1998) 541-546.

[2] BOALCH P., Stokes matrices, Poisson Lie groups and Frobenius manifolds, Invent. Math. 146 (2001) 479-506.

[3] Bondal A., A symplectic groupoid of triangular bilinear forms and the braid group, Preprint, 1999.

[4] Bondal A., Symplectic groupoids related to Poisson-Lie groups, Preprint, 1999.

[5] Cannas da Silva A., Weinstein A., Geometric Models for Noncommutative Algebras, in: Berkeley Mathematics Lecture Notes, Vol. 10, American Mathematical Society, Providence, RI, 1999.

[6] Courant T.J., Dirac manifolds, Trans. Amer. Math. Soc. 319 (1990) 631-661.

[7] CRAINIC M., Differentiable and algebroid cohomology, van Est isomorphisms, and characteristic classes, math.DG/0008064.

[8] DiRAC P.A.M., Lectures in Quantum Mechanics, Yeshiva University, 1964.

[9] DorfmAn I., Dirac Structures and Integrability of Nonlinear Evolution Equations, Wiley, Chichester, 1993.

[10] Drinfel'D V.G., On Poisson homogeneous spaces of Poisson-Lie groups, Theoret. Math. Phys. 95 (1993) 524-525.

[11] Dubrovin B., Geometry of 2D topological field theories, in: Integrable Systems and Quantum Groups, in: Lecture Notes in Math., Vol. 1620, Springer, Berlin, 1996, pp. 120-348.

[12] Etingof P., VARChenko A., Geometry and classification of solutions of the classical dynamical Yang-Baxter equation, Comm. Math. Phys. 192 (1998) 77-120.

[13] Felder G., Conformal field theory and integrable systems associated to elliptic curves, in: Proc. ICM Zürich, 1994, pp. 1247-1255.

[14] Fernandes R., Completely integrable bi-Hamiltonian systems, Ph.D. Thesis, University of Minnesota, 1994.

[15] Fernandes R., A note on Poisson symmetric spaces, in: Proceeding of the Cornelius Lanczos International Centenary Conference, 1994, pp. 638-642.

[16] Fernandes R., Connections in Poisson geometry I: Holonomy and invariants, J. Differential Geom. 54 (2000) 303-365.

[17] Fernandes R., Vanhaecke P., Hyperelliptic Prym varieties and integrable systems, Comm. Math. Phys. 221 (2001) 169-196.

[18] Ginzburg V., Weinstein A., Lie-Poisson structure on some Poisson Lie groups, J. Amer. Math. Soc. 5 (1992) 445-453.

[19] Li L.-C., PARMENTIER S., On dynamical Poisson groupoids I, math.DG/0209212.

[20] LiU Z.-J., Weinstein A., XU P., Manin triples for Lie bialgebroids, J. Differential Geom. 45 (1997) $547-574$.

[21] LiU Z.-J., Weinstein A., Xu P., Dirac structures and Poisson homogeneous spaces, Comm. Math. Phys. 192 (1998) 121-144. 
[22] LIU Z.-J., XU P., Exact Lie bialgebroids and Poisson groupoids, Geom. Funct. Anal. 6 (1996) 138145.

[23] LiU Z.-J., XU P., Dirac structures and dynamical $r$-matrices, Ann. Inst. Fourier 51 (2001) 831-859.

[24] LU J.H., Momentum mappings and reduction of Poisson actions, in: Symplectic Geometry, Groupoids, and Integrable Systems, MSRI Publ., Vol. 20, Springer, New York, 1991, pp. 209-226.

[25] Lu J.H., Weinstein A., Poisson Lie groups, dressing transformations, and Bruhat decompositions, J. Differential Geom. 31 (1990) 501-526.

[26] Lu J.H., Weinstein A., Groupoïdes symplectiques doubles des groupes de Lie-Poisson, C. R. Acad. Sci. Paris, Série I 309 (1989) 951-954.

[27] MackenzIE K., Xu P., Lie bialgebroids and Poisson groupoids, Duke Math. J. 18 (1994) 415-452.

[28] MackenzIE K., XU P., Integration of Lie bialgebroids, Topology 39 (2000) 445-467.

[29] Marsden J., Ratiu T., Reduction of Poisson manifolds, Lett. Math. Phys. 11 (1986) 161-169.

[30] Molino P., Structure transverse aux orbites de la représentation coadjointe : le cas des orbites réductives, Sém. Géom. Diff. USTL (Montpellier) (1984) 55-62.

[31] Richardson R.W., Springer T.A., The Bruhat order on symmetric varieties, Geom. Dedicata 35 (1990) 389-436.

[32] Springer T.A., Some results on algebraic groups with involutions, Adv. Stud. Pure Math. 6 (1985) 525-543.

[33] Ugaglia M., On a Poisson structure on the space of Stokes matrices, Internat. Math. Res. Notices 9 (1999) 473-493.

[34] Weinstein A., The local structure of Poisson manifolds, J. Differential Geom. 18 (1983) 523-557.

[35] Weinstein A., Symplectic groupoids and Poisson manifolds, Bull. Amer. Math. Soc. (N.S.) 16 (1987) 101-104.

[36] Weinstein A., The modular automorphism group of a Poisson manifold, J. Geom. Phys. 23 (1997) 379-394.

[37] XU P., Symplectic groupoids of reduced Poisson spaces, C. R. Acad. Sci. Paris, Série I 314 (1992) 457-461.

[38] XU P., On Poisson groupoids, Internat. J. Math. 6 (1995) 101-124.

(Manuscrit reçu le 26 octobre 2001; accepté, après révision, le 25 octobre 2002.)

Ping XU

Department of Mathematics, The Pennsylvania State University,

University Park, PA 16802, USA

E-mail: ping@math.psu.edu 